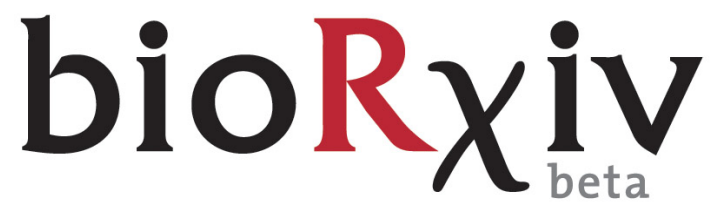

THE PREPRINT SERVER FOR BIOLOGY

\title{
Synthetic logic circuits using RNA aptamer against T7 RNA polymerase
}

Jongmin Kim, Juan F Quijano, Enoch Yeung, et al.

bioRxiv first posted online September 4, 2014

Access the most recent version at doi: http://dx.doi.org/10.1101/008771

Creative The copyright holder for this preprint is the author/funder. It is made available under Commons a CC-BY-ND 4.0 International license.

License 


\title{
Synthetic logic circuits using RNA aptamer against T7 RNA polymerase
}

\author{
Jongmin Kim ${ }^{1, \ddagger, \S, *}$, Juan F. Quijano ${ }^{2, \ddagger}$, Enoch Yeung ${ }^{3}$, Richard M. Murray ${ }^{1,3}$ \\ 1 Department of Biology and Biological Engineering, California Institute of Technology, \\ Pasadena, CA 91125, USA, 2 Licenciado en Biotecnología Genómica, Universidad Autónoma \\ de Nuevo León, Nuevo León, Mexico, 3 Department of Control and Dynamical Systems, \\ California Institute of Technology, Pasadena, CA 91125, USA \\ $\ddagger$ Equal contributions, $§$ Present address: Wyss Institute for Biologically Inspired Engineering, \\ Harvard University, Boston, MA 02115, USA \\ * Correspondence: jongmin@dna.caltech.edu
}

\begin{abstract}
Recent advances in nucleic acids engineering introduced several RNA-based regulatory components for synthetic gene circuits, expanding the toolsets to engineer organisms. In this work, we designed genetic circuits implementing an RNA aptamer previously described to have the capability of binding to the T7 RNA polymerase and inhibiting its activity in vitro. Using in vitro transcription assays, we first demonstrated the utility of the RNA aptamer in combination with programmable synthetic transcription networks. As a step to quickly assess the feasibility of aptamer functions in vivo, a cell-free expression system was used as a breadboard to emulate the in vivo conditions of E. coli. We tested the aptamer and its three sequence variants in the cell-free expression system, verifying the aptamer functionality in the cell-free testbed. In vivo expression of aptamer and its variants demonstrated control over GFP expression driven by T7 RNA polymerase with different response curves, indicating its ability to serve as building blocks for both logic circuits and transcriptional cascades. This work elucidates the potential of RNA-based regulators for cell programming with improved controllability leveraging the fast production and degradation time scales of RNA molecules.
\end{abstract}

Keywords: synthetic biology, logic circuits, cell-free breadboard, genelet, molecular programming, aptamer 


\section{Introduction}

Synthetic biology provides an engineering approach to the field of biology to (re)design tools and biological systems that can open up new possibilities in biotechnology and medicine $[1,2,3,4,5]$. To achieve this goal, such de novo biological systems need to be easily engineerable, ideally through utilizing molecular mechanisms that work much as the programming codes. The first synthetic gene networks, the toggle switch [6] and the repressilator [7], demonstrated the feasibility of programming computational tasks in living cells. Recent advances in synthetic biology further expanded the set of programs in biological organisms with novel functions including logic gates $[8,9,10,11,12]$, cell classifiers [13], and analog signal processors [14]. Most synthetic gene circuits constructed to date relied on well-characterized protein components, which results in a challenge for scaling up synthetic circuitry with predictable dynamical behaviors due to the idiosyncratic nature of each protein component involved. Recent advances aim to address such limitations through increasing the number of orthogonal protein regulators [15] as well as protein regulators that rely on nucleic acids as guiding molecules [16, 17].

To address the component bottleneck in synthetic biology, RNA structures have also been utilized as important tools for programming gene expression inspired by the naturally occuring nucleic-acid-based regulatory motifs $[18,19,20]$. By virtue of their predictive interactions with other nucleic acids, proteins, and small molecules, many RNA-based regulatory circuits have been investigated and synthesized $[21,22,23,24,25,26,27,28,29,30$, $31,32]$. Importantly, RNA aptamers possess the molecular recognition properties with specificity and affinity rivaling protein antibodies for exclusive ligands, allowing these molecules to form a useful set of components for synthetic circuitry in living cells. RNA aptamers are typically generated and selected in vitro starting from large random sequence libraries, and are subsequently optimized for high affinity binding to given ligands by a process known as SELEX (Systematic Evolution of Ligands by EXponential enrichment) [33, 34, 35, 36].

In a recent work, Ohuchi and co-workers [37] reported the in vitro evolution of RNA aptamers against T7 RNA polymerase (T7 RNAP) through SELEX. Although the detailed mechanism is not yet fully understood, it was shown that the RNA aptamers inhibited the association of T7 RNAP with T7 promoter DNA, thereby inhibiting the activity of RNAP in in vitro transcription assays. Utilizing the property of this aptamer, we implemented synthetic logic circuits in an in vitro transcription setting. As a step to quickly assess the aptamer functionality in vivo, a cell-free transcription-translation (TX-TL) system was employed as a 'breadboard' to emulate the in vivo conditions of $E$. coli for circuitry testing [38, 39]. Experimental results demonstrated that the T7 RNAP aptamer showed up to 8- and 5-fold inhibition in TX-TL system and in E. coli MGZ1 cells, respectively. Due to the wide-spread usage of T7 RNAP for in vitro and in vivo applications [40, 41, 42, 43], the T7 RNAP aptamer can be a useful addition to the regulatory toolkit for in vitro and in vivo synthetic biology.

\section{Materials and Methods}

Circuit construction and use of strains. Plasmid pAR1219 was obtained from Sigma-Aldrich. Plasmid pTara was provided by Matthew Bennett's group at Rice University. The rest of the circuits were constructed using Gibson assembly [44]. Schematics for plasmids are shown in the main text and the full sequences and annotations are described in Supplementary Figure S1. Plasmids were transformed into strains via chemical transformation. E. coli MGZ1 strain (MG1655 containing the LacI- and TetR-overexpressing Z1 cassette) was used for inducible expression from the TetR-regulated promoter. Plasmids pdeGFP-T7apt-4A, pdeGFP-T7apt-4G, and pdeGFP-T7apt- $4 \Delta$ were derived from the plasmid pdeGFPT7apt via round-the-horn site-directed mutagenesis using 5 '-phosphorylated primers for subsequent ligation. Plasmids containing the T7 RNAP aptamer and variants have an additional XhoI site between the aptamer and the terminator sequences (the functionality of the T7 RNAP aptamer was not affected by this feature). All of the plasmid sequences were confirmed by DNA sequencing.

In vitro transcription reaction. The sequence of all DNA molecules and expected RNA transcript sequences were chosen to minimize the occurrence of alternative secondary structures using the DNA and RNA folding program NUPACK [45]. The DNA and RNA sequences used in this study are listed in Supplementary material. All DNA oligonucleotides were purchased from Integrated DNA Technologies (USA). The T7 RNA polymerase (Cellscript, Madison, WI, USA; \#C-AS2607), T3 RNA polymerase (Promega, Madison, WI, USA; \#P4024), $10 \times$ transcription buffer and thermostable inorganic pyrophosphatase (New England Biolabs, Ipswich, MA, USA; \#B9012S, \#M0296S), NTP (Epicentre, Madison, WI, USA; \#RN02825) were purchased. Malachite green dye was purchased from Sigma (\#M9015). Since pyrophosphatase is involved in regulating the byproduct inorganic pyrophosphate for our transcriptional circuits and is not directly involved in the dynamics, we do not call it an "essential enzyme" for the circuit dynamics.

The fluorescence was recorded every minute using a Fluorolog-3 spectrofluorometer (Jobin Yvon, Edison, NJ, USA). The excitation and emission for malachite green fluorescence were at $630 \mathrm{~nm}$ and $655 \mathrm{~nm}$. DNA templates were annealed with $10 \%(\mathrm{v} / \mathrm{v}) 10 \times$ transcription buffer 
from $90^{\circ} \mathrm{C}$ to $20^{\circ} \mathrm{C}$ over 1 hour at $5 \mu \mathrm{M}$ concentrations. Transcription reactions were prepared by combining the annealed templates, $7.5 \mathrm{mM}$ of each NTP, $24 \mathrm{mM} \mathrm{MgCl}$ (to balance salt concentrations due to increased NTP concentrations), $10 \%(\mathrm{v} / \mathrm{v}) 10 \times$ transcription buffer, and $25 \mu \mathrm{M}$ malachite green dye in a test tube. Transcription reactions for spectrofluorometer experiments were prepared as a total volume of $60 \mu \mathrm{L}$ in quartz cuvettes and enzymes (RNAP and PPase) were added and mixed after the baseline fluorescence was recorded for $10 \mathrm{~min}$. Sample temperature was maintained at $29^{\circ} \mathrm{C}$ using a 4 -sample changer with a temperature-controlled water bath.

Media, chemicals and other reagents. Strains were grown in LB liquid medium or on agar plates $(1.5 \%$ agar), supplemented with carbenicillin $(100 \mu \mathrm{g} / \mathrm{ml})$, and chloramphenicol $(34 \mu \mathrm{g} / \mathrm{ml})$ as needed. IPTG induction was at $10 \mu \mathrm{M}$ unless otherwise noted. Anhydrotetracycline (aTc) induction experiments used different concentrations of aTc to achieve different levels of RNA aptamer expression as described in the figure captions.

Plate reader analysis. For cell-free TX-TL experiments, GFP fluorescence was measured using a filter set with excitation wavelength at $488 \mathrm{~nm}$ and emission wavelength at $507 \mathrm{~nm}$. All data were obtained using Perkin Elmer Victor X3 plate reader. Preparation of the cell-free TX-TL expression system was done according to previously described protocols [38, 39]. A single batch of $E$. coli extract was used consistently throughout the experiments to prevent variation from batch to batch. Samples were prepared by combining extract and buffer tubes freshly thawed from $-80^{\circ} \mathrm{C}$, and purified plasmid DNA. Concentrations of plasmids pAR1219 and pdeGFP-T7apt and its variants were $0.8 \mathrm{nM}$ for Figure 2. The concentrations of plasmids pAR1219, pT7-deGFP, and pTet-T7apt were $0.6 \mathrm{nM}$ for Supplementary Figure S3. The reaction volume was $10 \mu \mathrm{L}$ in a 384 -well plate (Nunc), and the number of replicates was two for each reaction. Sample temperature was maintained at $29^{\circ} \mathrm{C}$.

For in vivo experiments, cells were grown in shaking liquid culture (LB media with carbenillin and chloramphenicol) at $37^{\circ} \mathrm{C}$ overnight. Following this, the culture was diluted $1 / 200$ fold for plate reader measurements. GFP fluorescence was measured using a monochromater with excitation wavelength at $485 \mathrm{~nm}$ and emission wavelength at $525 \mathrm{~nm}$ in Biotek Synergy H1 plate reader. Fluorescence data were normalized by OD600 values. The culture volume was $200 \mu \mathrm{L}$ in a 96-well plate (Nunc), and the number of replicates was three for each condition. Sample temperature was maintained at $37^{\circ} \mathrm{C}$.

Flow cytometry analysis. Cells were grown in shaking liquid culture (LB media with carbenillin and chloramphenicol) at $37^{\circ} \mathrm{C}$ overnight. Following this, the culture was diluted $1 / 100$ fold and grown at $37^{\circ} \mathrm{C}(\mathrm{LB}$ media with antibiotics and $1 \%$ glucose and aTc) for two hours. Flow cytometry analysis used a Cell Lab Quanta SC MPL flow cytometer (Beckman Coulter). GFP was excited from a $488 \mathrm{~nm}$ laser and emission was measured from $517 \mathrm{~nm}$ longpass filter with a PMT setting of 5.0. For each sample, the GFP values of 50,000 cells were measured. Cells were then gated for GFP levels above background (2 a.u.). Data were analyzed using MATLAB and modal values were determined. The number of replicates was three for T7apt and the number of replicates were two for its variants T7apt-4A, T7apt-4G, and T7apt- $4 \Delta$.

\section{Results}

T7 RNAP aptamer for in vitro transcriptional circuits. First, we aim to demonstrate the utility of T7 RNAP aptamer for in vitro transcriptional circuits. Using the synthetic transcriptional switch as the regulatory motif and the aptamer for the chromophore malachite green (MG) as the output signal, we construct a logic circuit utilizing the property of T7 RNAP aptamer (Figure 1A). Among the several variants of T7 RNAP aptamers reported in Ohuchi et al. [37], our first in vitro assays utilized the aptamer T230-38. In this circuit, the two RNA polymerases, T3 RNAP and T7 RNAP, together with DNA activators serve as inputs and the fluorescence of MG aptamer serves as an output. Figure 1B illustrates the molecular reactions for this synthetic transcriptional circuit. An MG aptamer, MGapt, consists of a short RNA sequence whose central loop region serves as the binding pocket for MG. When MG is bound to the aptamer, it becomes highly fluorescent [46], thereby providing a convenient, real-time read-out for RNA expression. Previous in vitro synthetic transcriptional circuits utilized T7 RNAP [47, 48, 49, 50, 51]. However, since we aim to characterize an aptamer against T7 RNAP, a separate enzyme was required to drive the production of aptamer in order to avoid self-repression. Thus, we first verified the design principle for synthetic switches utilizing T3 RNAP using a template encoding MG aptamer with an incomplete promoter for T3 RNAP, pT3-MGapt; the production of MGapt was observed upon the addition of its cognate activator, input A2 (Supplementary Figure S2A). Utilizing the modular switch architecture where the output domain is physically separated from the input and promoter domains, the two templates were designed to transcribe different RNA outputs and to be recognized by two different RNA polymerases. (See Supplementary Method for sequences.) The two templates encoding T7apt and MGapt are hereby denoted as pT3T7apt and pT7-MGapt. In this circuit, T3 RNAP recognizes pT3 promoter upon addition of the DNA activator A2, and induces transcription of T7apt; T7 RNAP recognizes pT7 promoter upon addition of the DNA activator A1, and induces transcription of MGapt.

Experimentally, we initially included both templates 
A

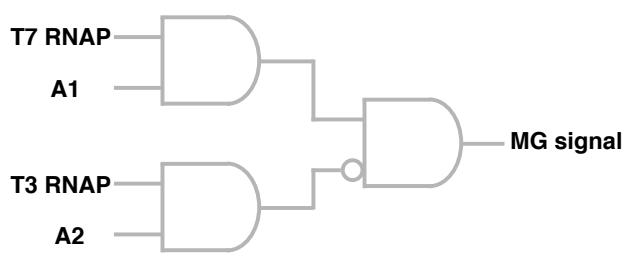

B

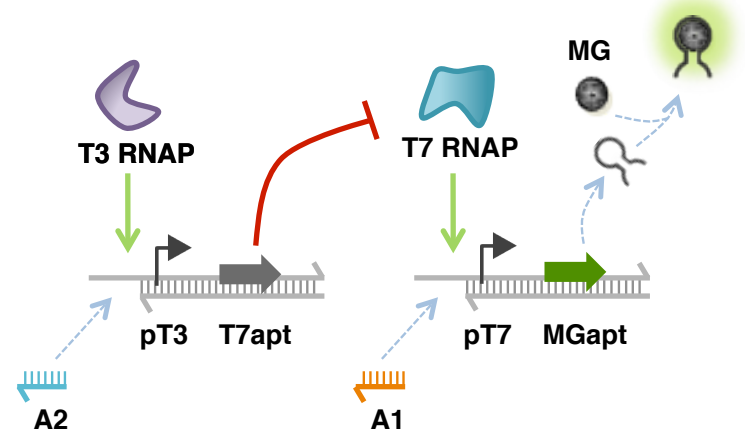

C

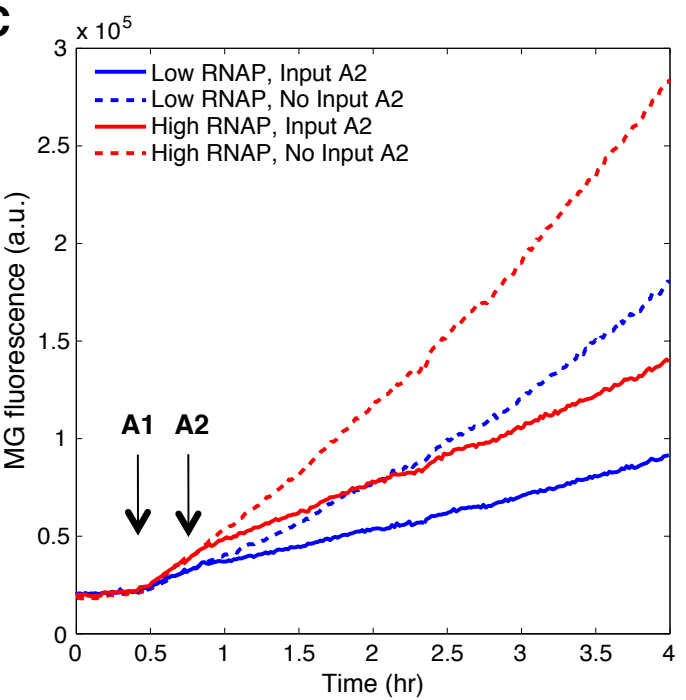

Figure 1: Characterization of T7 RNAP aptamer in in vitro transcription circuits. (A) Schematic representation of logic circuit. The two RNA polymerases and DNA activators serve as inputs and the fluorescence of malachite green aptamer serves as an ourput. (B) Detailed diagram of synthetic transcription circuit. Promoters pT3 and pT7 are to be recognized by T3 RNAP and T7 RNAP, respectively, upon addition of the corresponding DNA activator A2 and A1. The switch templates contain a promoter for RNA polymerase that lacks part of the double-stranded sequence; after addition of a single stranded DNA activator, the promoter is complete except for a nick, and thus, the transcription efficiency is high. When the MGapt is expressed, it can binds to the chemical compound malachite green, greatly increasing the fluorescence signal output. (C) Transcriptional assay of the circuit shown in (B). The two RNA polymerases, T3 RNAP and T7 RNAP, were added at $10 \mathrm{~min}$, the input DNA activator $\mathrm{A} 1$ was introduced at $25 \mathrm{~min}$ and the input DNA activator $\mathrm{A} 2$ was added at $45 \mathrm{~min}$ as marked by black arrows. The T7apt was efficient in repressing the T7 RNAP activity; about 3-fold decrease in MGapt signal was observed over 3 hours in the presence of T7apt. 'Low RNAP' and 'High RNAP' refer to T7 RNAP concentrations of 20 and $40 \mathrm{nM}$, respectively.

pT3-T7apt and pT7-MGapt, both T3 and T7 RNAP along with NTP fuel in the reaction mixture (Figure 1C). No MGapt expression was observed until the addition of DNA activator A1; upon the addition of input A1, the transcription of MGapt was immediately observed. Then, the DNA activator A2 was added for half of the samples, enabling transcription of T7apt in order to inhibit T7 RNAP-mediated MGapt expression (Figure 1C). The 'input A2' trajectories began to diverge from 'no input A2' trajectories about $10 \mathrm{~min}$ after the introduction of input A2 (Supplementary Figure S2B). The T7apt was efficient in repressing the T7 RNAP activity; about 3-fold decrease in MGapt signal was observed in the presence of T7apt. Further, we tested a modified T7apt termed T7apt-v2 where two bases comprising stem were switched and six additional nucleotides were attached at the $3^{\prime}$ end; 
T7apt-v2 was equally efficient in repressing the activity of T7 RNAP (Supplementary Figure S2C), indicating that the core structural motif of T7apt is sufficient for its activity.

T7 RNAP aptamer functionality in cell-free TX-TL system. After observing the T7 RNAP aptamer functionality as a circuit element in the synthetic transcription system, we designed a new circuit for in vivo application (Figure 2A and Supplementary Figure S3A). Creating a functional in vivo biocircuit can be a lengthy process, involving multiple iterations of design, assembly, and test cycles. Similar challenges may arise for testing novel regulatory mechanisms such as the inhibition of T7 RNAP through aptamers. To streamline and expedite the design-build-test cycles, we chose to employ cell-free TX-TL system; TX-TL system is gaining interest as biomolecular breadboards that take advantage of shorter assembly and troubleshooting times by conducting reactions in vitro emulating in vivo conditions [38, 39]. Because requirements for propagating plasmids in vivo have been removed, fast iterations of the design-assemblytest cycle of a given circuit is possible using linear DNA constructs [52]. Further, RNA-based regulatory elements were recently shown to behave analogously in TX-TL system when compared to in vivo, increasing the utility of this platform [53].

In order to emulate the T7 RNAP aptamer functioning in vivo, we assayed the aptamer in the TX-TL system. Initially, a three plasmid system was tested (Supplementary Figure S3B): plasmid pAR1219 encoding T7 RNAP under pLac promoter along with LacI constitutively expressed, plasmid pT7-deGFP encoding deGFP (truncated eGFP to maximize fluorescence output [38]) under pT7 promoter, and plasmid pTet-T7apt encoding T7 RNAP aptamer under pTet promoter. For a negative control, two plasmids pAR1219 and pT7-deGFP were introduced in TX-TL system in the absence of IPTG; for a positive control, the same plasmids were used in the presence of IPTG; the aptamer functionality was tested by including all three plasmids at the same concentrations with IPTG induction. The T7 RNAP aptamer was being expressed constitutively since TetR repressor was not present in TX-TL cell extract nor was it encoded on the plasmids used. Eight-fold reduction of deGFP output was obtained in the presence of the plasmid expressing the $\mathrm{T} 7$ RNAP aptamer (Supplementary Figure S3C).

After the initial verification of T7 RNAP aptamer activity in TX-TL, the expression cassettes contained in the plasmids pT7-deGFP and pTet-T7apt were joined to create the plasmid pdeGFP-T7apt (Figure 2A). The replication origin and antibiotic resistance was also switched for pdeGFP-T7apt since we aim to investigate its in vivo activity. Also, mutant variants of the T7 RNAP aptamer were constructed through site-directed mutagenesis in or- der to disrupt the conserved sequence domain presumably responsible for T7 RNAP inhibition [37]. The purpose of mutant constructs was two-fold: first, to demonstrate that T7 RNAP inhibition was not mediated by the resource exhaustion caused by T7 RNAP aptamer overproduction [54] and second, to verify the functional importance of conserved sequence domain. The nucleotides 28-31 of the T7apt sequence was targeted and replaced by AAAA, GGGG, or deleted, resulting in three aptamer variants: pdeGFP-T7apt-4A, pdeGFP-T7apt-4G, and pdeGFP-T7apt- $4 \Delta$ (Figure 2C).

These new set of plasmids were tested together with pAR1219 in the TX-TL expression system (Figure 2B). The results in the TX-TL system were as expected: the correct T7 RNAP aptamer (T7apt) showed 8-fold inhibition of the GFP output as in the previous experiment, whereas the mutant aptamers showed at most 2-fold inhibition, indicating that the core structure of the aptamer was presumably disrupted as a result of mutation. The GFP expression was observed after about $1 \mathrm{hr}$ of TX-TL reaction possibly due to slow production and folding of T7 RNAP, and soon afterwards the slope of GFP production showed clear distinction depending on the identity of input aptamer used. Notably, the GFP output reduction was less than 1.5 fold for T7apt- $4 \Delta$; it is plausible that overexpression of non-specific RNA species could cause a small reduction in GFP expression due to resource usage [54].

Characterization of T7 RNAP aptamer circuit in vivo. Encouraged by the activity of T7 RNAP aptamer in the cell-free TX-TL system, we carried out the characterization of circuit in vivo, utilizing $E$. coli MGZ1 strain containing constitutively expressed LacI and TetR to complete the inducible circuit. In this circuit, IPTG will induce the production of T7 RNAP, hence driving the expression of deGFP, while aTc will induce the production of T7 RNAP aptamer, repressing the expression of deGFP (Figure 3A). The fluorescence measurement in plate reader clearly showed that aTc-induced T7apt expression inhibited the expression of deGFP (Figure 3B). When T7apt expression was induced with high concentration of aTc $(0.6 \mu \mathrm{g} / \mathrm{mL}), 5$-fold reduction in the deGFP output was obtained compared to no aTc. In contrast, all the mutant variants when induced by the same concentration of aTc showed no effect on the deGFP output (Figure 3B), indicating that the inhibition of T7 RNAP activity is mediated by the correct aptamer rather than by cellular stress due to aTc or overexpression of non-specific RNA species. The fluorescence output decreased once the cells reach stationary phase, however, the fold-change was reasonably consistent over time (Supplementary Figure S4A). Similar results were obtained when $E$. coli were grown in LB or LB with $10 \mu \mathrm{M}$ IPTG although the foldchange was reduced to 2.5 fold and 2 fold, respectively 
A
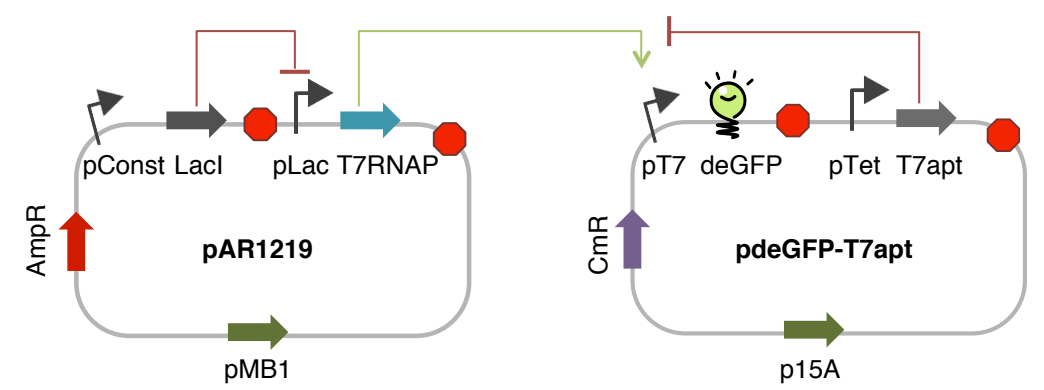

B

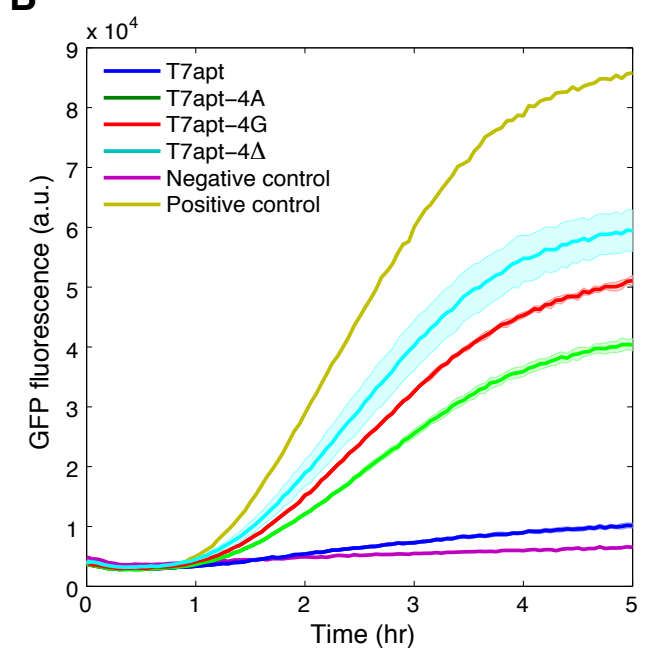

C

plasmid

pdeGFP-T7apt pdeGFP-T7apt-4A pdeGFP-T7apt-4G pdeGFP-T7apt- $4 \Delta$

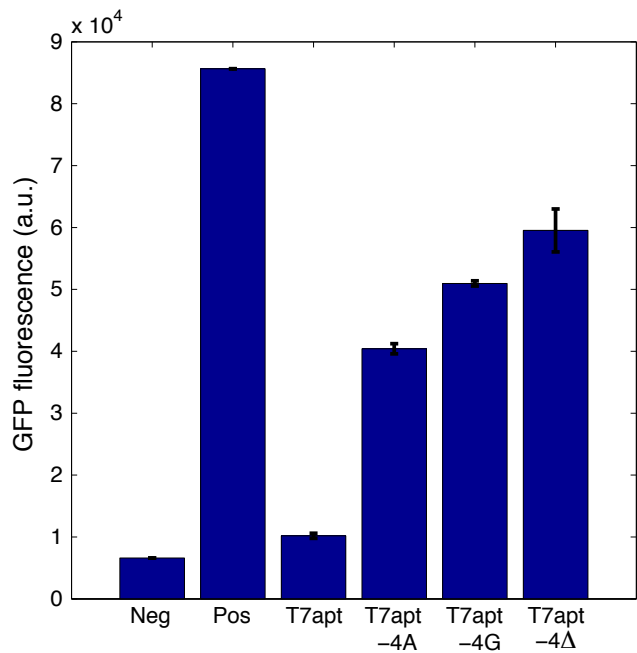

aptamer sequence
GGCGAGCGUAAGUCAAUUCCACUAUCAUUGCUGCAAGC GGCGAGCGUAAGUCAAUUCCACUAUCAAAAAUGCAAGC GGCGAGCGUAAGUCAAUUCCACUAUCAGGGGUGCAAGC GGCGAGCGUAAGUCAAUUCCACUAUCA_UGCAAGC

Figure 2: Characterization of T7 RNAP aptamer and its variants in the cell-free TX-TL system. (A) Two-plasmid system used for aptamer-mediated T7 RNAP inhibition test in the TX-TL cell-free extract. (B) The expression of T7 RNAP was induced by IPTG and the effect of expression of T7 RNAP aptamer and its variants was tested in TX-TL system. TX-TL results showed that deGFP expression was approximately 8-fold decreased in the presence of the correct aptamer; the fluorescence signals were much higher in the presence of modified aptamers indicating disrupted functionality. Negative control contained only pAR1219 plasmid and positive control contained pAR1219 and pT7-deGFP plasmids. The T7 RNAP aptamer production was not inhibited by TetR since the cell extract does not contain TetR protein. The fluorescence signal from deGFP was measured at $5 \mathrm{hrs}$ for the bar plot. (C) The sequence of T7 RNAP aptamer and its variants. Red color highlights the modified consensus sequence domain.

(Supplementary Figure S4BC).

A more detailed characterization of the circuit was carried out by testing the response of the circuit to five different IPTG concentrations ranging from 0 to a $30 \mu \mathrm{M}$ and six different aTc concentrations ranging from 0 to 0.6 $\mu \mathrm{g} / \mathrm{mL}$. As shown in Figure 3B, the response is characterized by high GFP expression for high IPTG concentra- tions and low aTc concentrations. (The response curves are shown in Supplementary Figure 4D.) At high concentration of IPTG $(30 \mu \mathrm{M})$, the response was marked by a constant high GFP expression, possibly due to overexpression of T7 RNAP beyond that of T7apt. Even higher concentration of IPTG $(100 \mu \mathrm{M})$ or aTc $(1 \mu \mathrm{g} / \mathrm{mL})$ severely impaired the cell growth, and therefore, those 


\section{A}
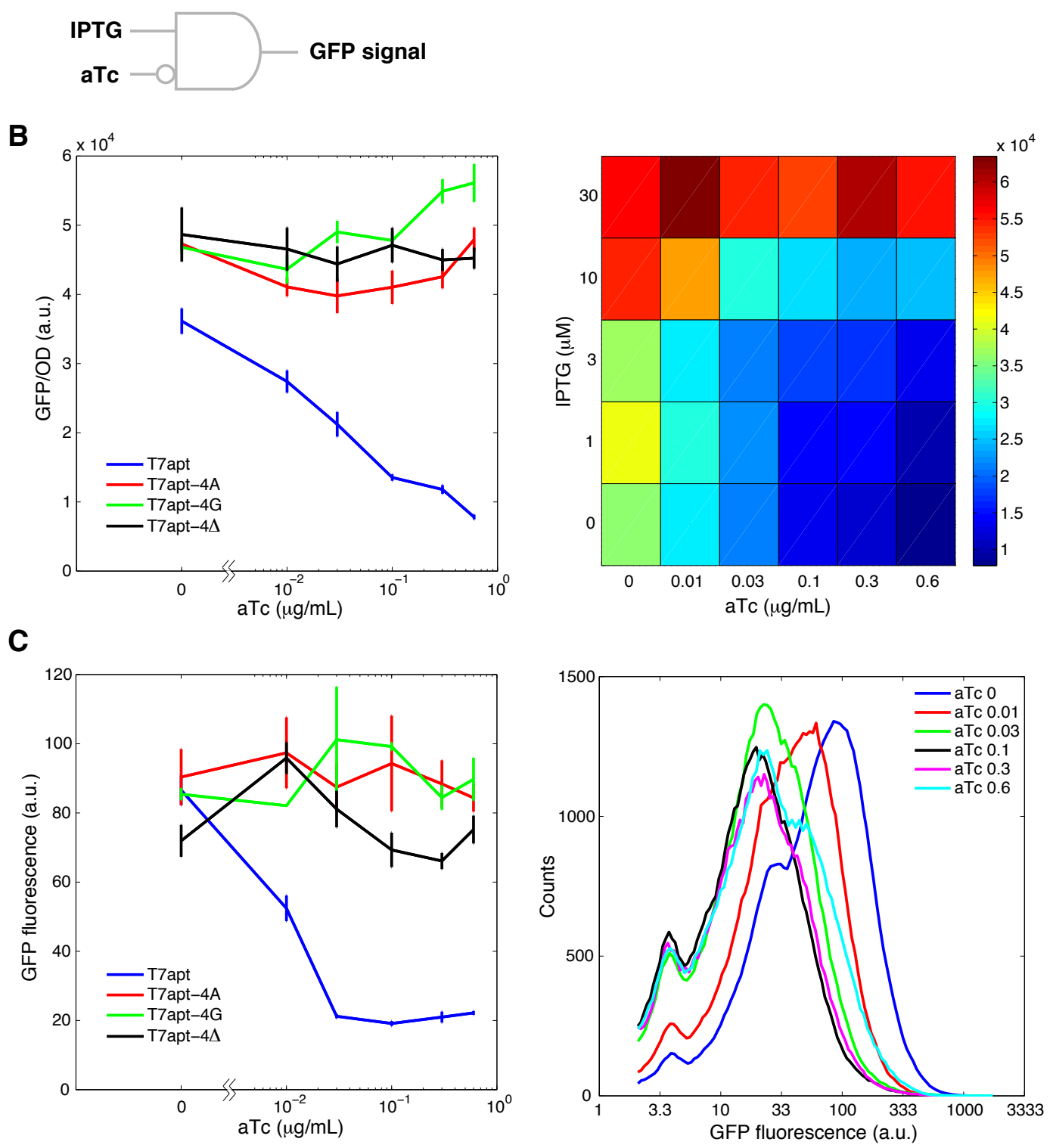

Figure 3: Characterization of T7 RNAP aptamer and its variants in vivo. E. coli MGZ1 containing constitutively expressed Lacl and TetR was used with two plasmids pAR1219 and pdeGFP-T7apt and its variants as shown in Figure 2A to complete the inducible circuit. (A) Circuit diagram of T7 RNAP aptamer in vivo. Inputs are small molecules IPTG and aTc and the output is GFP fluorescence. (B) Plate reader analysis for the response curve of T7 RNAP aptamer and its variants upon aTc induction. GFP/OD values in LB/1\% glucose media were measured at $8 \mathrm{hrs}$ for the plot. Time courses are shown in Supplementary Figure S4A. The GFP/OD value heat map for different levels of aTc and IPTG inputs is shown on the right. (C) Flow cytometry analysis for the response curve of T7 RNAP aptamer and its variants upon aTc induction. The modal values are plotted with the standard deviation of replicate measurements as error bars. One of the replicate GFP histogram (50,000 events) for T7apt is shown on the right.

regions were not further explored. Overall, the circuit exhibited a logic gate behavior as shown in Figure 3A.

To explore the circuit response at the individual cell levels, flow cytometry analysis was performed. Repre- sentative flow cytometry histograms of GFP output for different aTc inducer levels clearly showed that GFP output is decreased upon induction of T7apt expression (Figure $3 \mathrm{C}$ ). The modal fluorescence values from these his- 
tograms were used to plot the response curves for T7apt and its variants. The GFP expression showed no dependence on aTc induction levels for mutant T7apt (Figure 3C and Supplementary Figure S5), confirming the findings of plate reader analysis.

\section{Discussion}

Synthetic gene circuitry and cellular programming open up new opportunities for biotechnological and medical applications. With the growing appreciation for RNA molecules as the key component of genetic regulatory networks, efforts to utilize the programmable structure and function of RNA molecules for synthetic biology have accelerated in the recent past. The demonstration of functional RNA aptamer in living cells provides an alternative to simple transcription regulatory circuits with transcription factors, expanding the toolkit to program cells in synthetic biology.

In this work, we show that the T7 RNAP aptamer has the capability of inhibiting the T7 RNAP acivity in vitro and in vivo with a predictable functional behavior as a modular component within synthetic network. We first demonstrated the activity of T7 RNAP aptamer in in vitro transcription circuits. It is not surprising that the aptamer would be functional since the assay environment is similar to the previous works [37]; however, we provide a framework for systematic circuit construction utilizing other RNAP and aptamer components - T3 RNAP and MG aptamer. It remained a question whether T7 RNAP aptamer can show functionality in vivo given the complexity of in vivo environment with potentially strong crosstalk and degradation for aptamer. We utilized a cell-free TX-TL system as a testbed to quickly assess the feasibility of our approach in vivo. Despite certain limitations [54], TX-TL system provides a solid expression platform to connect in vitro activity to in vivo functionality $[38,52]$, as recently demonstrated for synthetic RNA circuitry [53]. We used equal concentrations of plasmids for TX-TL experiment to correlate with the plasmids that have pMB1 and p15A origins with similar medium copy numbers - this may partly explain the similar results observed in the aptamer-mediated T7 RNAP repression in vitro and in vivo $(\approx 8$-fold vs. $\approx 5$-fold repression). Together with growing appreciation of the need for testbed in synthetic biology $[55,56,57]$, the detailed characterization of cell-free expression platform may provide an easy-to-use breadboard to expedite implementation of synthetic biology circuits in vivo. For in vivo characterization, E. coli MGZ1 cells displayed reasonably high deGFP output even without IPTG induction - this could be due to a weak repression of T7 RNAP expression or a leakage from the medium-copy plasmid, considering that even a small amount of T7 RNAP is sufficient to direct high-level transcription from a T7 promoter. Nevertheless, when cells were induced with IPTG, higher deGFP output was obtained, and repression was observed as expected when induced with aTc (except when IPTG induction was very strong, possibly exceeding the expression level of T7 RNAP aptamer). Utilizing a stronger inducible promoter and improving stability of RNA aptamer using tRNA scaffold [58] or hairpins [59] may increase the dynamic range provided by T7 RNAP aptamer.

A number of straightforward extensions could be implemented for a broad application of the T7 RNAP aptamer. Synthetic circuitry with another aptamer against RNAP, e.g., an aptamer against SP6 RNAP [60], could be utilized to design a synthetic bistable system or oscillators $[61,62]$. It is also possible to utilize an antisense signal for T7 RNAP aptamer to produce adaptation behavior [51, 63]. Although widely adapted for gene expression in vivo, T7 RNAP due to its very strong transcriptional activity can cause growth arrest and toxicity when strongly induced. Analogous to recent studies utilizing RNA aptamers for quantitation of mRNA [54, 64], concatenation of T7 RNAP aptamer at the $3^{\prime}$ end of an mRNA driven by $\mathrm{T} 7$ promoter can provide a necessary negative feedback to regulate the expression levels of target genes, thereby reducing toxicity upon T7 RNAP induction in vivo. An intriguing possibility that remains to be tested is whether the T7 RNAP aptamer can provide differential regulation of recently reported synthetic T7 RNAP variants with different promoter specificity $[42$, 43].

\section{Supplementary data}

Supplementary Data are available.

\section{Funding}

This work was supported by National Science Foundation award no. 0832824 (The Molecular Programming Project) and the Defense Advanced Research Projects Agency (DARPA/MTO) Living Foundries program, contract number HR0011-12-C-0065 (DARPA/CMO). The views and conclusions contained in this document are those of the authors and should not be interpreted as representing officially policies, either expressly or implied, of the Defense Advanced Research Projects Agency or the U.S. Government.

\section{Acknowlegments}

The authors thank David Shis and Matthew Bennett for providing the pTara plasmid.

Conflict of interest statement. None declared. 


\section{References}

[1] Sprinzak, D. and Elowitz, M. B. (2005) Reconstruction of genetic circuits. Nature, 438, 443-448.

[2] Purnick, P. E. M. and Weiss, R. (2009) The second wave of synthetic biology: from modules to systems. Nat Rev Mol Cell Biol, 10, 410-422.

[3] Khalil, A. S. and Collins, J. J. (2010) Synthetic biology: applications come of age. Nat. Rev. Genet., 11, 367-379.

[4] Weber, W. and Fussenegger, M. (2009) The impact of synthetic biology on drug discovery. Drug Discov Today, 14, 956-963.

[5] Gibson, D. G., et al. (2010) Creation of a bacterial cell controlled by a chemically synthesized genome. Science, $\mathbf{3 2 9}, 52-$ 56 .

[6] Gardner, T. S., Cantor, C. R., and Collins, J. J. (2000) Construction of a genetic toggle switch in Escherichia coli. Nature, 403, 339-342.

[7] Elowitz, M. B. and Leibler, S. (2000) A synthetic oscillatory network of transcriptional regulators. Nature, 403, 335-338.

[8] Moon, T. S., Lou, C., Tamsir, A., Stanton, B. C., and Voigt, C. A. (2012) Genetic programs constructed from layered logic gates in single cells. Nature, 491, 249-253.

[9] Siuti, P., Yazbek, J., and Lu, T. K. (2013) Synthetic circuits integrating logic and memory in living cells. Nat Biotechnol, 31, 448-452.

[10] Win, M. N. and Smolke, C. D. (2008) Higher-order cellular information processing with synthetic RNA devices. Science, 322, 456-460.

[11] Ausländer, S., Ausländer, D., Müller, M., Wieland, M., and Fussenegger, M. (2012) Programmable single-cell mammalian biocomputers. Nature, 487, 123-127.

[12] Bonnet, J., Yin, P., Ortiz, M. E., Subsoontorn, P., and Endy, D. (2013) Amplifying genetic logic gates. Science, 340, 599603.

[13] Xie, Z., Wroblewska, L., Prochazka, L., Weiss, R., and Benenson, Y. (2011) Multi-input RNAi-based logic circuit for identification of specific cancer cells. Science, 333, 1307-1311.

[14] Daniel, R., Rubens, J. R., Sarpeshkar, R., and Lu, T. K. (2013) Synthetic analog computation in living cells. Nature, 497, 619623.

[15] Stanton, B. C., Nielsen, A. A. K., Tamsir, A., Clancy, K., Peterson, T., and Voigt, C. A. (2014) Genomic mining of prokaryotic repressors for orthogonal logic gates. Nat Chem Biol, 10, 99-105.

[16] Qi, L. S., Larson, M. H., Gilbert, L. A., Doudna, J. A., Weissman, J. S., Arkin, A. P., and Lim, W. A. (2013) Repurposing CRISPR as an RNA-guided platform for sequence-specific control of gene expression. Cell, 152, 1173-1183.

[17] Bikard, D., Jiang, W., Samai, P., Hochschild, A., Zhang, F., and Marraffini, L. A. (2013) Programmable repression and activation of bacterial gene expression using an engineered CRISPR-Cas system. Nucleic Acids Res, 41, 7429-7437.

[18] Brantl, S. and Wagner, E. G. H. (2000) Antisense RNAmediated transcriptional attenuation: an in vitro study of plasmid pT181. Mol Microbiol, 35, 1469-1482.

[19] Gultyaev, A. P., Franch, T., and Gerdes, K. (1997) Programmed cell death by hok/sok of plasmid R1: Coupled nucleotide covariations reveal a phylogenetically conserved folding pathway in the hok family of mRNAs. J Mol Biol, 273, 26-37.
[20] Winkler, W., Nahvi, A., and Breaker, R. R. (2002) Thiamine derivatives bind messenger RNAs directly to regulate bacterial gene expression. Nature, 419, 952-956.

[21] Bayer, T. S. and Smolke, C. D. (2005) Programmable ligandcontrolled riboregulators of eukaryotic gene expression. Nature Biotech., 23, 337-343.

[22] Buskirk, A. R., Kehayova, P. D., Landrigan, A., and Liu, D. R. (2003) In vivo evolution of an RNA-based transcriptional activator. Chem Biol, 10, 533-540.

[23] Desai, S. K. and Gallivan, J. P. (2004) Genetic screens and selections for small molecules based on a synthetic riboswitch that activates protein translation. J Am Chem Soc, 126, $13247-13254$.

[24] Grate, D. and Wilson, C. (2001) Inducible regulation of the $S$. cerevisiae cell cycle mediated by an RNA aptamer-ligand complex. Bioorg Med Chem, 9, 2565-2570.

[25] Isaacs, F. J., Dwyer, D. J., Ding, C., Pervouchine, D. D., Cantor, C. R., and Collins, J. J. (2004) Engineered riboregulators enable post-transcriptional control of gene expression. Nature Biotech., 22, 841-847.

[26] Saha, S., Ansari, A. Z., Jarrell, K. A., and Ptashne, M. (2003) RNA sequences that work as transcriptional activating regions. Nucleic Acids Res, 31, 1565-1570.

[27] Suess, B., Fink, B., Berens, C., Stentz, R., and Hillen, W. (2004) A theophylline responsive riboswitch based on helix slipping controls gene expression in vivo. Nucleic Acids Res, 32, 1610-1614.

[28] Werstuck, G. and Green, M. R. (1998) Controlling gene expression in living cells through small molecule-RNA interactions. Science, 282, 296-298.

[29] Yen, L., Svendsen, J., Lee, J.-S., Gray, J. T., Magnier, M., Baba, T., D'Amato, R. J., and Mulligan, R. C. (2004) Exogenous control of mammalian gene expression through modulation of RNA self-cleavage. Nature, 431, 471-476.

[30] Win, M. N. and Smolke, C. D. (2007) A modular and extensible RNA-based gene-regulatory platform for engineering cellular function. Proc. Natl. Acad. Sci. USA, 104, 14283-14288.

[31] Callura, J. M., Cantor, C. R., and Collins, J. J. (2012) Genetic switchboard for synthetic biology applications. Proc Natl Acad Sci USA, 109, 5850-5855.

[32] Mutalik, V. K., Qi, L., Guimaraes, J. C., Lucks, J. B., and Arkin, A. P. (2012) Rationally designed families of orthogonal RNA regulators of translation. Nat Chem Biol, 8, 447-454.

[33] Ellington, A. D. and Szostak, J. W. (1990) In vitro selection of RNA molecules that bind specific ligands. Nature, 346, 818822 .

[34] Stoltenburg, R., Reinemann, C., and Strehlitz, B. (2007) SELEX-a (r)evolutionary method to generate high-affinity nucleic acid ligands. Biomol Eng, 24, 381-403.

[35] Tuerk, C. and Gold, L. (1990) Systematic evolution of ligands by exponential enrichment: RNA ligands to bacteriophage T4 DNA polymerase. Science, 249, 505-510.

[36] Vinkenborg, J. L., Karnowski, N., and Famulok, M. (2011) Aptamers for allosteric regulation. Nat Chem Biol, 7, 519527.

[37] Ohuchi, S., Mori, Y., and Nakamura, Y. (2012) Evolution of an inhibitory RNA aptamer against T7 RNA polymerase. FEBS Open Bio, 2, 203-207.

[38] Shin, J. and Noireaux, V. (2012) An E. coli cell-free expression toolbox: Application to synthetic gene circuits and artificial cells. ACS Synth. Biol., 1, 29-41. 
[39] Sun, Z. Z., Hayes, C. A., Shin, J., Caschera, F., Murray, R. M., and Noireaux, V. (2013) Protocols for implementing an escherichia coli based TX-TL cell-free expression system for synthetic biology. J Vis Exp, p. doi: 10.3791/50762.

[40] Milligan, J. F. and Uhlenbeck, O. C. (1989) Synthesis of small RNAs using T7 RNA polymerase. Methods Enzymol, 180, 5162 .

[41] Fuerst, T. R., Niles, E. G., Studier, F. W., and Moss, B. (1986) Eukaryotic transient-expression system based on recombinant vaccinia virus that synthesizes bacteriophage T7 RNA polymerase. Proc Natl Acad Sci USA, 83, 8122-8126.

[42] Temme, K., Hill, R., Segall-Shapiro, T. H., Moser, F., and Voigt, C. A. (2012) Modular control of multiple pathways using engineered orthogonal T7 polymerases. Nucleic Acids Res, 40, 8773-8781.

[43] Ellefson, J. W., Meyer, A. J., Hughes, R. A., Cannon, J. R., Brodbelt, J. S., and Ellington, A. D. (2014) Directed evolution of genetic parts and circuits by compartmentalized partnered replication. Nat Biotechnol, 32, 97-101.

[44] Gibson, D. G., Young, L., Chuang, R.-Y., Venter, J. C., III, C. A. H., and Smith, H. O. (2009) Enzymatic assembly of dna molecules up to several hundred kilobases. Nat Methods, 6 , 343-345.

[45] Zadeh, J. N., Steenberg, C. D., Bois, J. S., R.Wolfe, B., Pierce, M. B., Khan, A. R., Dirks, R. M., and Pierce, N. A. (2011) NUPACK: Analysis and design of nucleic acid systems. J Comput Chem, 32, 170-173.

[46] Grate, D. and Wilson, C. (1999) Laser-mediated, site-specific inactivation of RNA transcripts. Proc Natl Acad Sci USA, 96, 6131-6136.

[47] Kim, J., White, K. S., and Winfree, E. (2006) Construction of an in vitro bistable circuit from synthetic transcriptional switches. Mol. Syst. Biol., 2, 68.

[48] Kim, J. and Winfree, E. (2011) Synthetic in vitro transcriptional oscillators. Mol. Syst. Biol., 7, 465.

[49] Franco, E., Friedrichs, E., Kim, J., Jungmann, R., Murray, R., Winfree, E., and Simmel, F. C. (2011) Timing molecular motion and production with a synthetic transcriptional clock. Proc. Natl. Acad. Sci. USA, 108, E784-E793.

[50] Weitz, M., Kim, J., Kapsner, K., Winfree, E., Franco, E., and Simmel, F. C. (2014) Diversity in the dynamical behaviour of a compartmentalized programmable biochemical oscillator. Nat Chem, 6, 295-302.

[51] Kim, J., Khetarpal, I., Sen, S., and Murray, R. M. (2014) Synthetic circuit for exact adaptation and fold-change detection. Nucleic Acids Res, 42, 6078-6089.

[52] Sun, Z. Z., Yeung, E., Hayes, C. A., Noireaux, V., and Murray, R. M. (2014) Linear DNA for rapid prototyping of synthetic biological circuits in an Escherichia coli based TX-TL cell-free system. ACS Synth Biol, 3, 387-397.

[53] Takahashi, M. K., et al. (2014) Rapidly characterizing the fast dynamics of RNA genetic circuitry with cell-free transcriptiontranslation (TX-TL) systems. ACS Synth Biol.

[54] Siegal-Gaskins, D., Tuza, Z. A., Kim, J., Noireaux, V., and Murray, R. M. (2014) Gene circuit performance characterization and resource usage in a cell-free "breadboard". ACS Synth Biol, 3, 416-425.

[55] Hodgman, C. E. and Jewett, M. C. (2012) Cell-free synthetic biology: thinking outside the cell. Metab. Eng., 14, 261-269.

[56] Shimizu, Y., Inoue, A., Tomari, Y., Suzuki, T., Yokogawa, T., Nishikawa, K., and Ueda, T. (2001) Cell-free translation reconstituted with purified components. Nature Biotech., 19, $751-755$.
[57] Chappell, J., Jensen, K., and Freemont, P. S. (2013) Validation of an entirely in vitro approach for rapid prototyping of DNA regulatory elements for synthetic biology. Nucleic Acids Res, 41, 3471-3481.

[58] Paige, J. S., Wu, K. Y., and Jaffrey, S. R. (2011) RNA mimics of green fluorescent protein. Science, 333, 642-646.

[59] Carrier, T. A. and Keasling, J. D. (1999) Library of synthetic $5^{\prime}$ secondary structures to manipulate mRNA stability in Escherichia coli. Biotechnol Prog, 15, 58-64.

[60] Mori, Y., Nakamura, Y., and Ohuchi, S. (2012) Inhibitory RNA aptamer against SP6 RNA polymerase. Biochem Biophys Res Commun, 420, 440-443.

[61] Mardanlou, V., Tran, C. H., and Franco, E. (2014) Design of a molecular bistable system with RNA-mediated regulation. IEEE Conference on Decision and Control $(C D C)$.

[62] Blanchini, F., Samaniego, C. C., Franco, E., and Giordano, G. (2014) Design of a molecular clock with RNA-mediated regulation. IEEE Conference on Decision and Control (CDC).

[63] Franco, E., Giordano, G., Forsberg, P.-O., and Murray, R. M. (2014) Negative autoregulation matches production and demand in synthetic transcriptional networks. ACS Synth Biol, 3, 589-599.

[64] Pothoulakis, G., Ceroni, F., Reeve, B., and Ellis, T. (2013) The spinach RNA aptamer as a characterization tool for synthetic biology. ACS Synth Biol, 3, 182-187. 


\section{Supplementary Information Methods}

The sequence of DNA oligonucleotides and RNA outputs used for in vitro transcription experiments are listed as follows.

\section{DNA sequences}

pT3-T7apt-nt (77mer), 5'-AAGCAAGGGTAAGATGGAATGAAATTAACCCTCACTAAAGGCGAGCGTAAGTCAATTCCACTATCATTGCTGCAAGC- ${ }^{\prime}$.

pT3-T7apt-t (49mer), 5'-GCTTGCAGCAATGATAGTGGAATTGACTTACGCTCGCCTTTAGTGAGGG-3'.

pT3-T7apt-v2-nt (83mer), 5' -AAGCAAGGGTAAGATGGAATGAAATTAACCCTCACTAAAGGCGAGCGTAAGTCAATTCCACTATCATTGCTGCTTGCCTCGAG-3'.

pT3-T7apt-v2-t (55mer), 5'-CTCGAGGCAAGCAGCAATGATAGTGGAATTGACTTACGCTCGCCTTTAGTGAGGG-3' .

pT3-MGapt-nt (84mer), 5' -AAGCAAGGGTAAGATGGAATGAAATTAACCCTCACTAAAGGACAAGCATCCCGACTGGCGAGAGCCAGGTAACGAATGGATGCG-3'.

pT3-MGapt-t (56mer), 5'-CGCATCCATTCGTTACCTGGCTCTCGCCAGTCGGGATGCTTGTCCTTTAGTGAGGG-3' pT7-MGapt-nt (84mer), 5'-CTAATGAACTACTACTACACACTAATACGACTCACTATAGGACAAGCATCCCGACTGGCGAGAGCCAGGTAAC-GAATGGATGCG-3'

pT7-MGapt-t (57mer), 5'-CGCATCCATTCGTTACCTGGCTCTCGCCAGTCGGGATGCTTGTCCTATAGTGAGTCG-3' .

A2-T3 (36mer), 5'-TTAATTTCATTCCATCTTACCCTTGCTTCAATCCGT-3'.

A1-T7 (35mer), 5'-TATTAGTGTGTAGTAGTAGTTCATTAGTGTCGTTC-3'

\section{RNA output sequences}

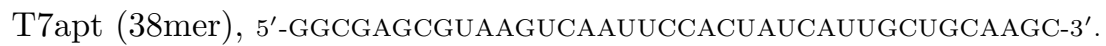

T7apt-v2 (44mer), 5'-GGCGaGCGUAAGUCAAUUCCACUAUCAUUGCUGCUUGCCUCGAG- ${ }^{\prime}$.

MGapt (45mer), 5'-GGACAAGCAUCCCGACUGGCGAGAGCCAGGUAACGAAUGGAUGCG-3' .

The sequence of DNA oligonucleotides used for cloning and Gibson assembly are listed as follows.

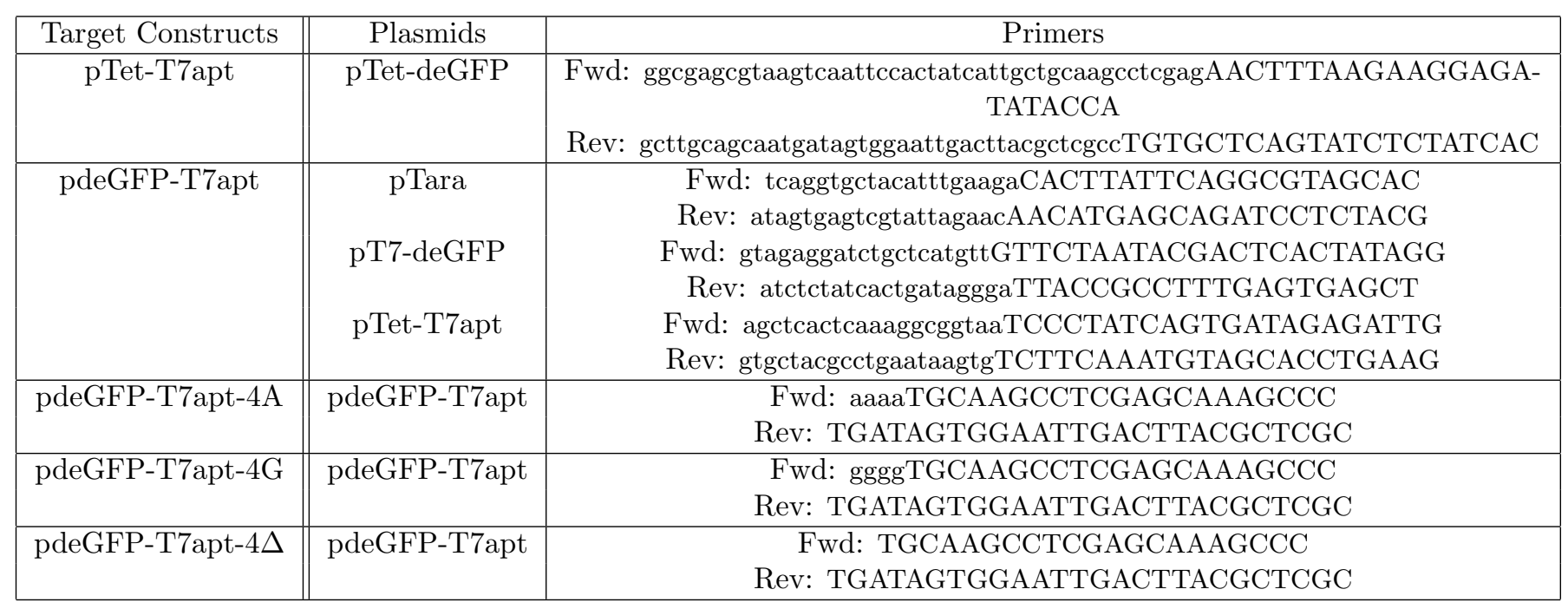

* Lower case letters indicate the parts of sequence that are different from the starting plasmids. For pTet-T7apt plasmid, XhoI digestion and subsequent ligation was performed to remove deGFP sequence. For pdeGFP-T7apt variant plamids, primers were $5^{\prime}$-phosphorylated and a subsequent quick ligation step was performed. 


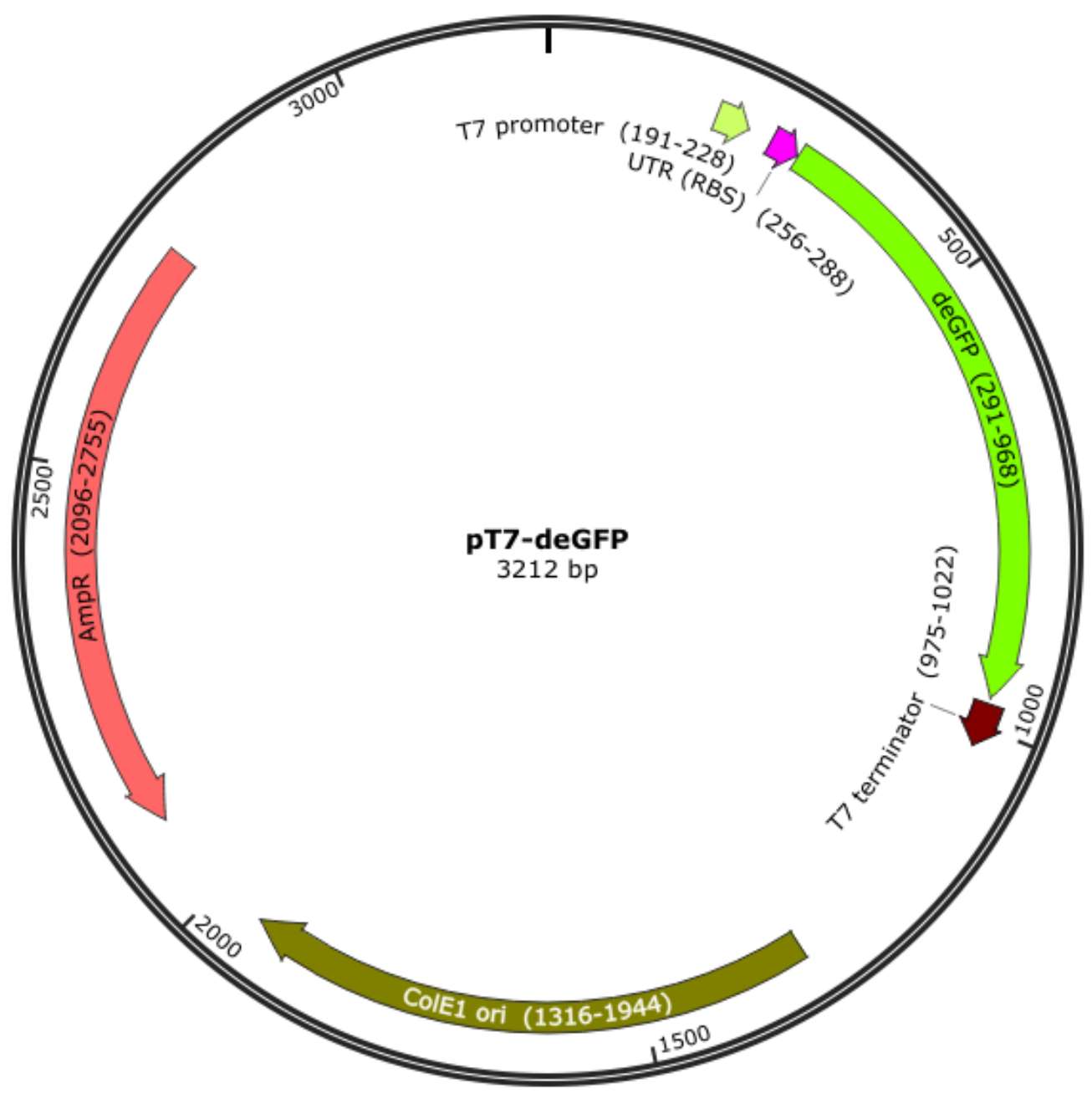

01

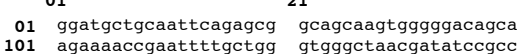

201

301 TCACTGGCGTGTTCCCATC

401 GCTGACCCTGAAGTTCATCI

$\mathbf{5 0 1}$ CCCGACCACATGAAGCAGC.

$\mathbf{6 0 1}$ CCCGCGCCGAGGTGAAGTTC

$\mathbf{7 0 1}$ GGAGTACAACTACAACAGCC

$\mathbf{8 0 1}$ GCAAAGACCCCAACGAGAA

901 acgggtcttgaggggtttt

1101 ttatgactgtctctetatc

1202 ggcgagcggtatcagctc

1401 CCGACAGGACTATAAAGAT

1501 TCCCTTCGGGAAGCGTGGCG

1601 CGTTCAGCCCGACCGCTGC

1701 ATTAGCAGAGCGAGGTATG

1901 GCGCAAPACCIICGA

2001 ttatcaaaaagatcttCaC

2101 GCTTAATCAGTGAGGCACC

2201 ACCATCTGGCCCCAGTGCT

2301 AGTGGTCCTGCAACTITA

2501 CAMCCACAGCANC

2601 CTMATCTCATCCAMCC

2701 CGGCGTCAATACGGGATAA

2801 GCTGTTGAGATCCAGTTCG

2901 CAAAATGCCGCAAAAAAGG

3001 tcatgagcggatacatatt

3101 tattatcatgaca
41 ggcgagagccag

CCACACGCCCAACCACCCC GCACCACCGGCAAGCTGCC GAGGGCGACACCCTEGTGAA acAacGTCtatatcatgGcC CGCCAGCAGAACACCCCCA tggtcgaccgatgccettga atgcaactcgtaggacaggt tcaaaggcggtaatacggt CCAGGCGTTTCCCCCTGGAA CTITCTCAATGCTCACGCTG TITATCCGGTAACTATCGT AGGCGGTGCTACAGAGTTCT AAGAAGATCCTTTGATCTT ctagatccttttaaattaaa CAATGATACCGCGAGACCA CGCCTCCATCCAGTCTATTA AAGATGCTTTTCTGTGACTG ACCGCGCCACATAGCAGAAC MAACCCACTCGTGCACC gaatgtatttagaaaataa ataaaataggcgtatcacg GCTCACGCTCGTCGTTTGG gaagacctgaccgccgcaga tgatgcgtgaacgtgacgga gtaacgaatggatcCAATAA COIAAACGECCACAAGITCA TGCCCGAAGGCTACGTCCAO CCGCATCGAGCTGAAGGGCA GACAAGCAGAAGAACGCCAT TCGECGACEGCCCCGTCCTE GAGTTCGTGACCGCCGCCS gagccttcaacccagtcagc gecgcagogotctecgct atccacagaatcaggggata GCTCCCTCGTGCGCTCTCCT TAGGTATCTCAGTTCGGTGT CTTGAGTCCAACCCGGTAAG TGAAGTGGTGGCCTAACTAC TCTACg aatgaagttttaaatcaatc TCGIICATCCATAGTTGCC CGCTCACCGGCTCCAGATTI ATAGC GTCAGAAGTAAGTTGGCCGC GTGAGTACTCAACCAAGTCA TTTAAAAGTGCTCATCATTG GTTGAATACTCATACtCt acaaataggggttccgcgca cattccccgaaaagtgcca aggcccttcotcttcaaga attctggcgaatcctctga
81

tggatgtttgacatggtga agactatcgcaccatcagcc gtaaccaccgcgacatgtg tgtgctgttc

GTIGTITAACTTMAAGAAG GAGATATACCATGGAGCTTI OACCACCCTGACCTACGGCG GECGATECCACCTACEGCAA AGCGCACCATCTTCTTCAA GGACGACGGCAACTACAAGA

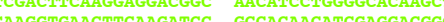
CIGCCCGACAMCCA CPACC

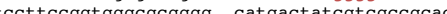
tcctcgctcactgactcgct gcgctcggtcgttcggctgc gactega gcasa GCATCACAAAAATCGACGCT CAAGTCAGAGGTGGCGAAC GTTCCGACCCTGCCGCTTAC CGGATACCTGTCCGCCTTTC AGGTCGTTCGCTCCAAGCTG GGCTGTGTGCACGAACCCCC ACACGACTTATCGCCACTGG CAGCAGCCACTGGTAACAGG GGCTACACTA gtggaacgaaaactcacgtt aagggattttggtcatgaga taagtatatatgagtaaac ttggtctgacag

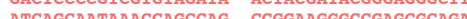
a cCGgaAgGgCceagcGCaga manccajacto J TTCTTAAAATAGT GCGACCGAGTTGCTCTTTGCC GAAAACGTTC TACTTTCACCAGCGTTTCTE GGTGAGCAAAAACAGGAAG 


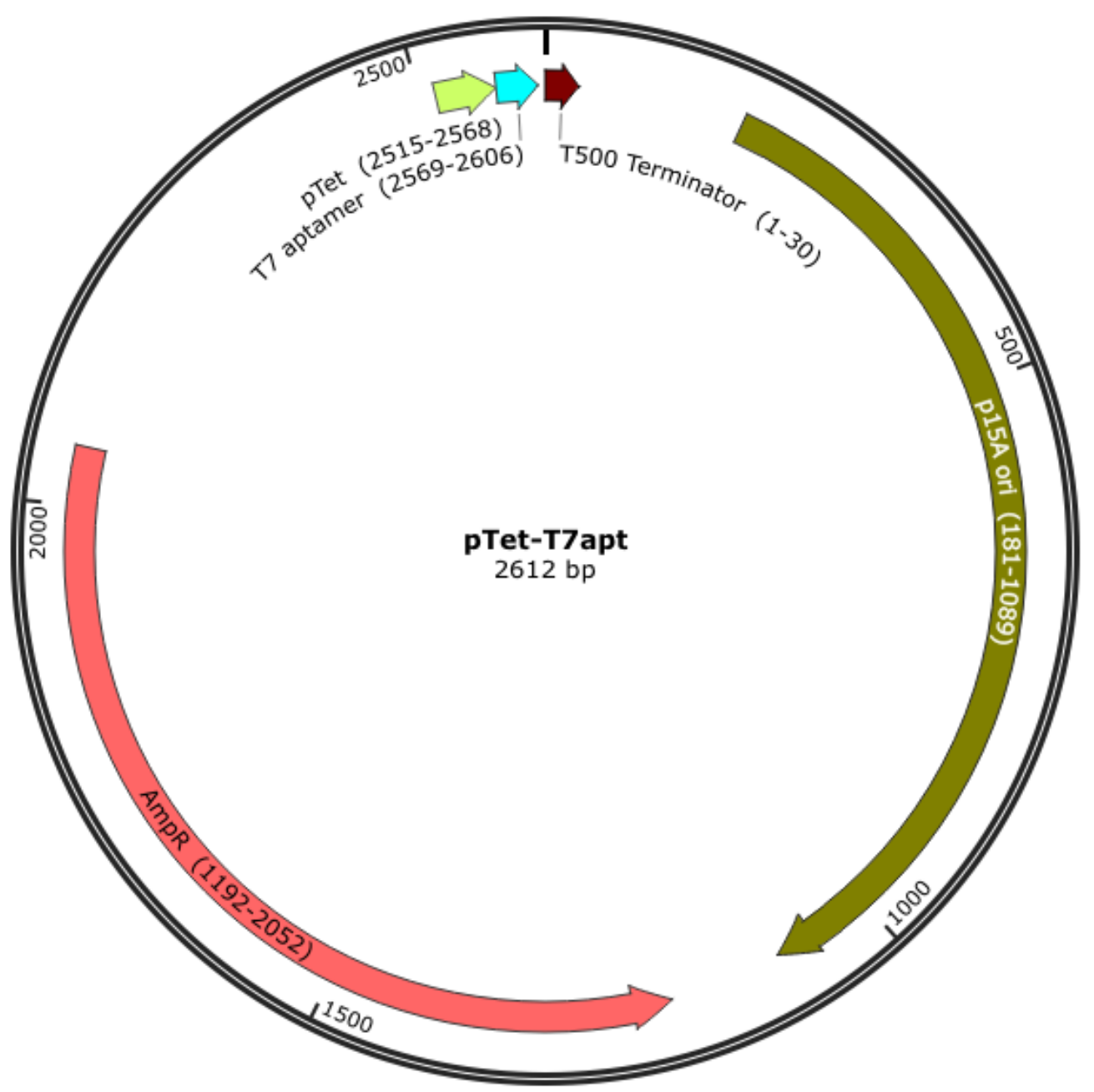

01

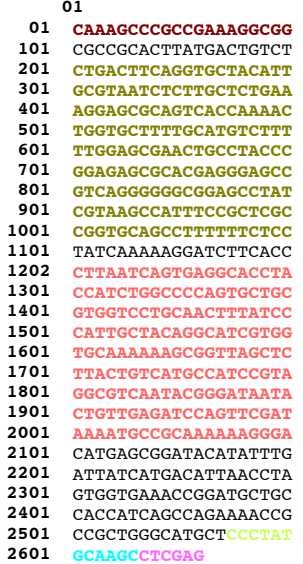

21

GCTTTTCTGTGTCGACCGAT TCTTTTATCATGCAACTCGTA TGAAGAGATAAATTGCACTC AACGAAAAAACCGCCTTGCA TTGTCCTTTCAGTTTAGCC CCGGGTTGGACTCAAGACGA GGAACTGAGTGTCAGGCGTG GCCAGGGGAAACGCCTGGT CGCAGTCGAACGACCGAGC TGCCACATGAAGCACTTCA TAGATCCTTTTAAAATTAAA TCTCAGCGATCTGTCTATT AATGATACCGCGAGACCCA GCCTCCATCCAGTCTATTA TGTCACGCTCGTCGTTTGG CTTCGGTCCTCCGATCGTTG AGATGCTTTTCTGTGACTGG CCGCGCCACATAGCAGAAC: GTAACCCACTCGTGCACCC ATAAGGGCGACACGGAAAT AATGTATTTAGAAAAATAAA AATTCAGAGCGGCAGCAAGT AATTTTGCTGGGTGGGCTAA GGGGGACAGCAGAAGACCTG
CGATATCCGCCTGATGCGTG 41 GGACAGGTGCCGGCATGATA AAATCTAGAAATATTTTATC GGGCGGTTTTTCGAAGGTTC TAACCGGCGCATGACTTCAA TAGTTACCGGATAAGGCGCA TCTTTATAGTCCTGTCGGGT GCCCTCTCACTTCCCTGTTA TAGCGAGTCAGTGAGCGA TGACACCCTCATCAGTGCCA ATGAAGTTTTAAATCAATC GCTCACCGGCTCCAGATTTA TTGTTGCCGGGAAGCTAGAG ATGGCTICATICAGCTCCGG TCAGAAGTAAGTTGGCCGCA TGAGTACTCAACCAAGTCAT ACTGATCTTCAGCATCTIT TTGAATACTCATACTCTTCC CAAATAGGGGTTCCGCGCAC GGGGGACAGCAGAAGACCTG 61

CAGTCAGCTCCTTCCGGTGG GCGCGGGGCATGACTATCGT AGCTGTCAAACATGAGAATT ACAACTTATATCGTATGGGG TGATTAATAAGATGATCTTC TTGAGATCGTTTTGGTCTGC ICTGAGCTACCAACTCTTTG AACCGAGGTAACTGGCTTGG gactaactcctctaaatca tTaccagtgectectgccag GCGGTCGACTGAACGGGGG GTTCGTGCATACAGTCCAGC TAAACCGAAGGCAGGAACA

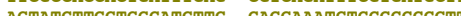
magcega ACATAGTA AAAGTATATATGAGTAAACT TCGTCTCACAGTTACCHATE ACTCCCCCTCGTGTACATAA CTACGATACGGGAGGGCMTI TCAGCAATAAACCAGCCAGC CGGAAGGGCCGAGCGCAGA TAAGTAGTTCGCCAGTTAAT AGTTTGCGCAACGTTGTTGC TTCCCAACGATCAAGGCGAG TTACATGATCCCCCATGTTG GTGTTATCACTCATGGTTAT GGCAGCACTGCATAATTCTC ACTGAGAATAGTGTATGCGG CGACCGAGTTGCTCTTGCCC AAAACGITCITCGGGGGAA AACTCTCAAGGATCTIACC ACT GTGACAAAACAGGAAGGC ATTATCAGgGtTATtGTC: DTU МСCGCCGAGAGTCGATGIT TGACATGGTGAAGACTATCG

Figure S1: Plasmid maps. Plasmid pT7-deGFP was derived from the plasmid pIVEX2.3d (Roche) [38]. Plasmid pTetT7apt was constructed using Gibson assembly method with plasmid pBEST-Luc (Promega) as a backbone. Plasmid pTara was used as a backbone to join pT7-deGFP and pTet-aptamer expression cassettes in plasmid pdeGFP-T7apt. 


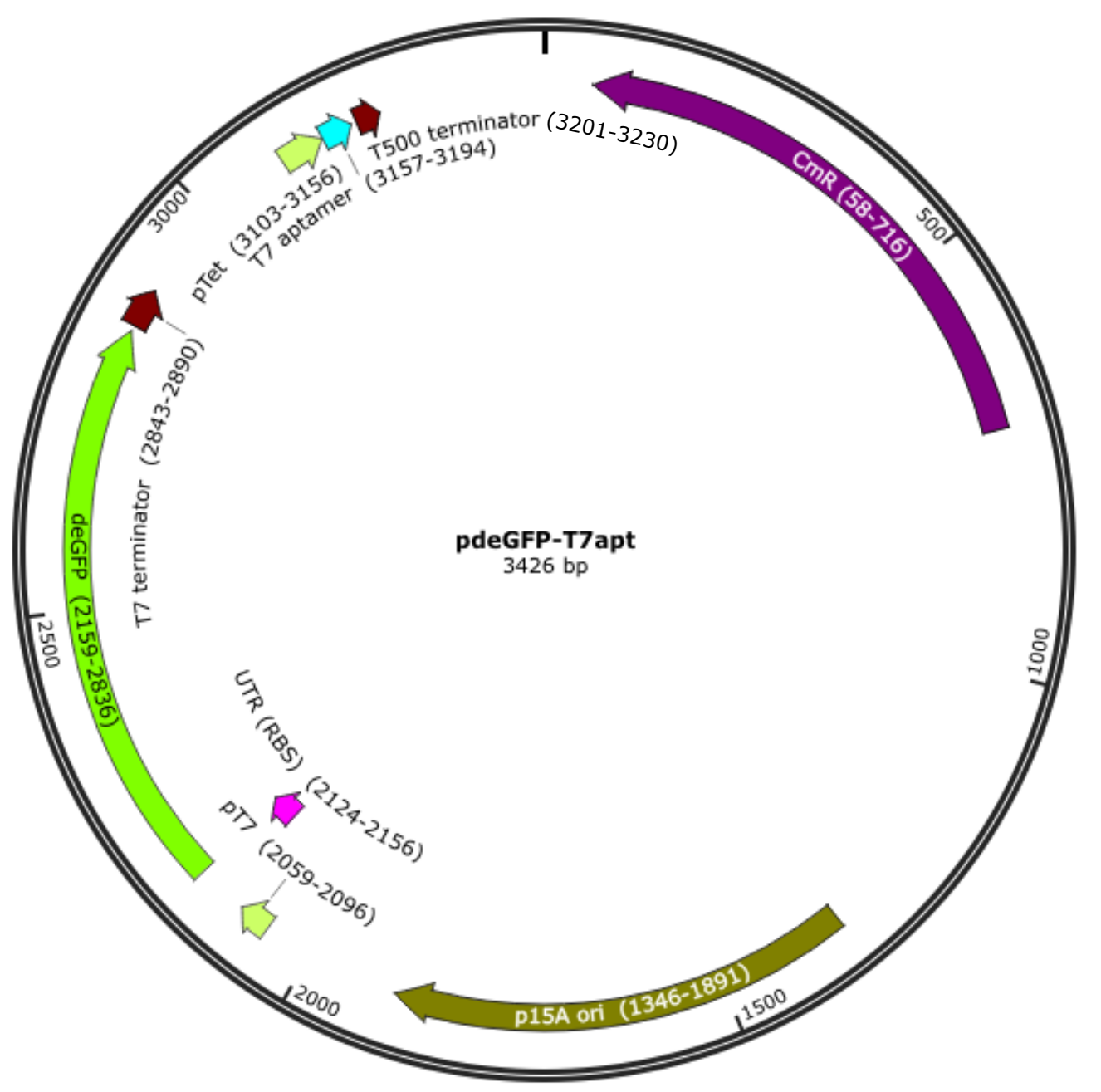

01

01 cacttattcaggcgtagcac

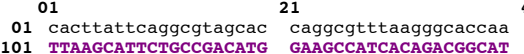

201 TGGTGAATCTCCGACATG GAAGCCATCACAGACGGCA

301 AATAAACCCTTTAGGGAAA

401 CAGAGCGATGAAAACGTTIC

501 ATTCCGGATGAGCATTCATC

601 CAGCTGAACGTTCTGGTTAT

801 aacctcttacgtgcogatca

901 atcttccgtcacaggtatt

1101 cttactatgttggcactga

$\mathbf{1 1 0 1}$ cttactatgttggcactgat

1301 ggaagatacttaacaggga

1401 TGGTGGCGAAACCCGACAG

1601 AGTCCGACCGCTGCGCCTT

1701 AGGAGTTAGTCTTGAAGTC

1801 GGTAGCTCAGAGAACCTTC

1901 cttattaatcagataaaat

2101 agagccaggtaacgaatgg

2301 AGCTGCCCETECCCTECCC

2401 GTCCGCCATGCCCGAAGCC

2501 CTGGTGAACCGCATCGAGC

$\mathbf{2 6 0 1}$ TCATGGCCGACAAGCAGAR

2801 GTCCTCCTEGA ETTTCETG?

2901 gccettgagagcettcaacc

3001 ggacaggtgceggcagcgc

3101 atc

3201 CAAAGCCCGCCGAAAGGCG

3301 cgccgcacttatgactgtc ACCTGTCCGCO ACCСTCETGACCACCCTGAC CGTCCAGGAGCGCACCAT GAAGGGCATCGACTTCAAGG AACGGCATCAAGGTGAACT CGCCGCCGGGATCTAACtcg cagtcagctccttccggtgg ttccgcttcctcgctcac 作 AAAGGCGGATAAAACTTGT GCTTATTTTTCTITACGGTC TGAAATGCCTCAAAATGTTC TTTACGATGCCATTGGGATA a attgeccagggcttccog tatcaacagggacaccagga cctcctgttcagctactgac ggggtggtgcgtaacggcaa aagcaccgccggacatcagc gagggtgtcagtgaagtgct tcatgtggcaggagaaaaa ggctgcaccggtgcgtcagc ttcgactgcggcgagcggaa atggcttacgaacggggcgg gtgagagggccgcggcaaag ccgttTTTCCATAGGCTCCG CCCCCCTGACAAGCATCACG (TCCTGGCGGCTCCCTC TTGTCTCATTCCACGCCTGA CACTCAGTTCCGGGTAGGCA TCCGGTAACTATCGTCTTGA GTCCAACCCGGAAAGACAT AAAAACCGCCCTGCAAGGCG GTTTTTTCGTTTTCAGAGCA acgatgcgtccggcgtagag gatctgctcatgttgttc tCCAATAATMTTITAACT TTAAGAAGGAGATATACC

CTTTCTGTgtcgaccgat gccettgagagcettcaa tgaaga
81 CGCAGTACTGTTGTAATTCA TTGCGTATAATATTTECCCA AAACGAAAAACATATTCTC AATCGTCGTGGTATTCACTC gicto TTTAAAAAGGCCGTAATATC TATATCA ttcattatggtgaaagttgg ttttgaggtctgcgaagtg gctagcggagtgtatactgg agaatatgtgatacaggata agatttcctggaagatgcca AAATCTGACGCTCAAACA CTITCGGT G TATGCACGAACCCCCCGTTC A GCCACTGGTAATTGATTTAG TIACCICGGITCAAAGAGTT A AACATCTCAAgaagatcat ggcgccagcaaccgcacctg

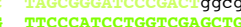

TTCCCATCCTGETCGAGCTE GITCACTOCACCACCGCCA AAGCACCACGACTICTICAA TGAATTCAGGCGACACC GCCGACCACTACCAGCACAA ACGAGAAGCGCARTCACATC GGGTTTTTTGgtcgaccgat tctttatcatgcaactcgta cagctcactcaaaggcggta g gegcggggcatgactatcgt acaacttatatcgtatgggg 
A

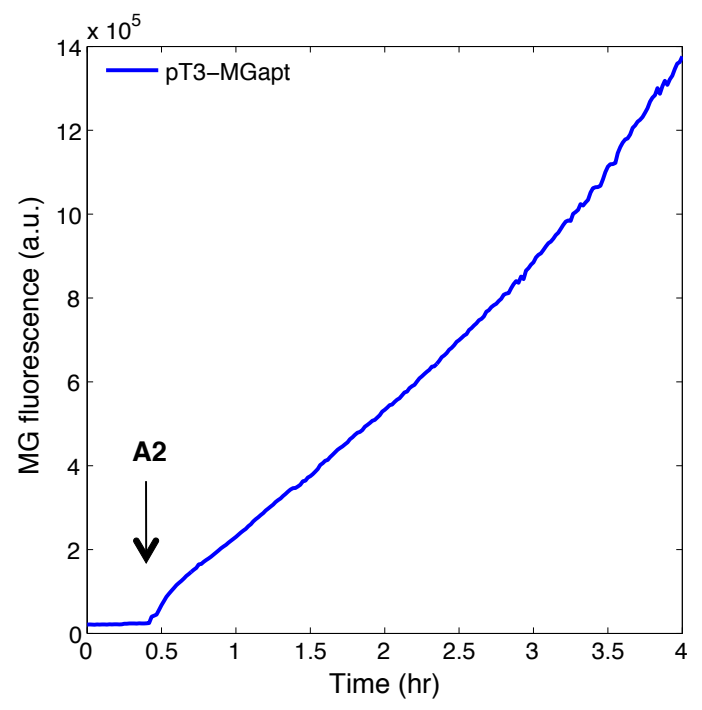

C

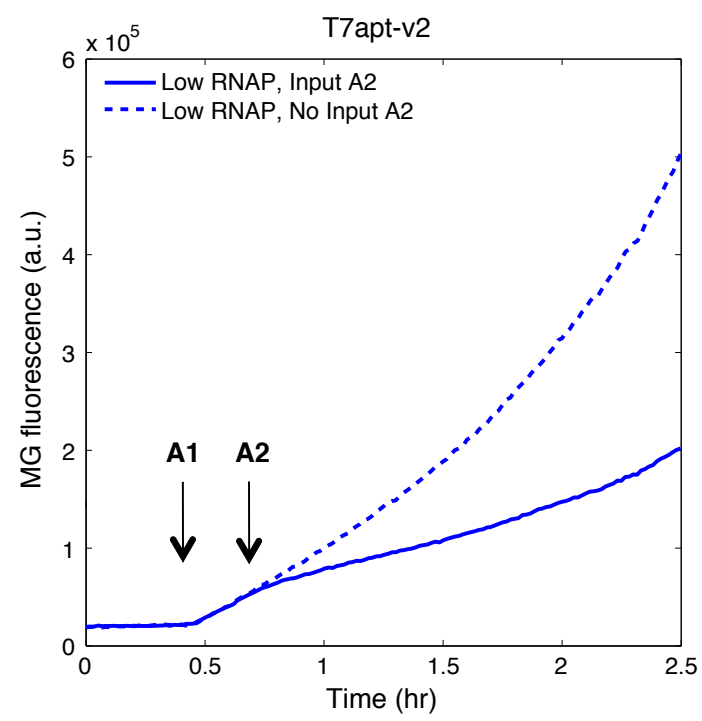

B
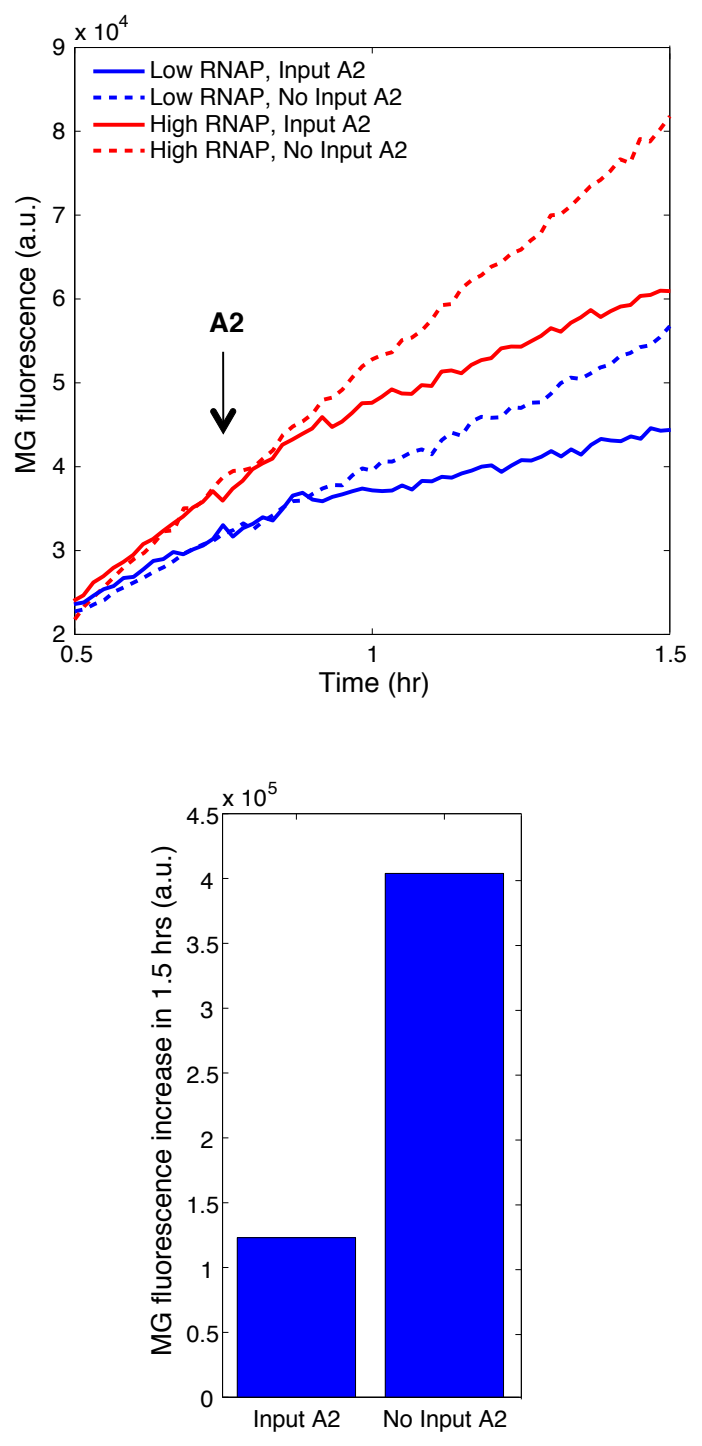

Figure S2: Spectrofluorometer analysis of T7 RNAP aptamer in in vitro transcription circuits. (A) With pT3-MGapt template in a transcription buffer in a cuvette, T3 RNAP was added at $10 \mathrm{~min}$. MG aptamer was not produced until the activator A2 was added at $25 \mathrm{~min}$, indicating that the incomplete promoter design also works for T3 promoter much as T7 promoter design used for genelet circuits. (B) Close-up of the timecourse experiment shown in Figure 1C. The activator A2 was added at $40 \mathrm{~min}$. The 'input A2' trajectories began to diverge from 'no input A2' trajectories about 10 min after the introduction of input A2. (C) Test of modified aptamer against T7 RNAP (T7apt-v2). Here, pT3-T7apt-v2 template was used instead of pT3-T7apt. The two RNA polymerases were added at $10 \mathrm{~min}$, the input A1 was added at $25 \mathrm{~min}$, and the input A2 was added at 45 min following the protocols used in Figure 1C. The T7apt-v2 was efficient in repressing the T7 RNAP activity; about 3-fold decrease in MGapt signal was observed over 1.5 hours in the presence of the T7apt-v2. 
A

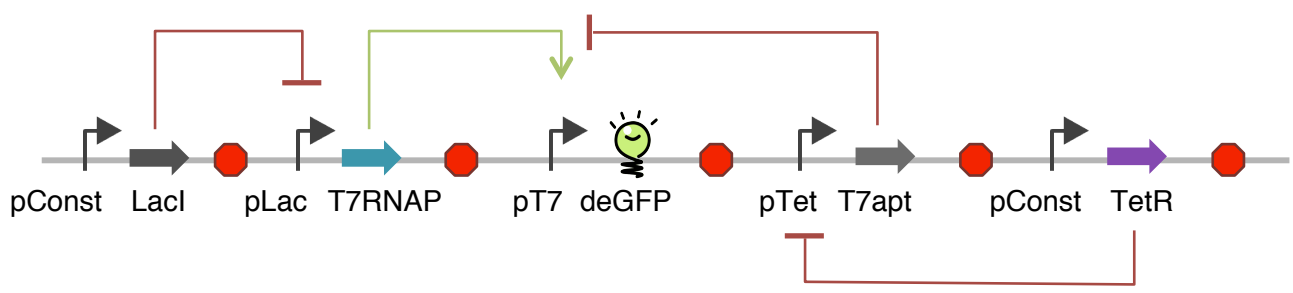

B

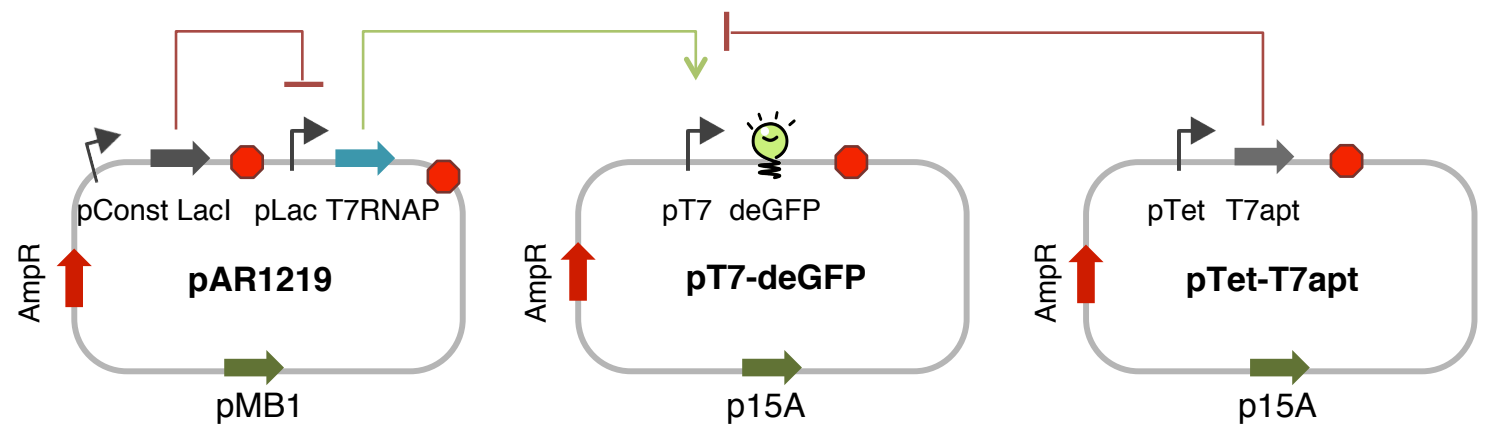

C

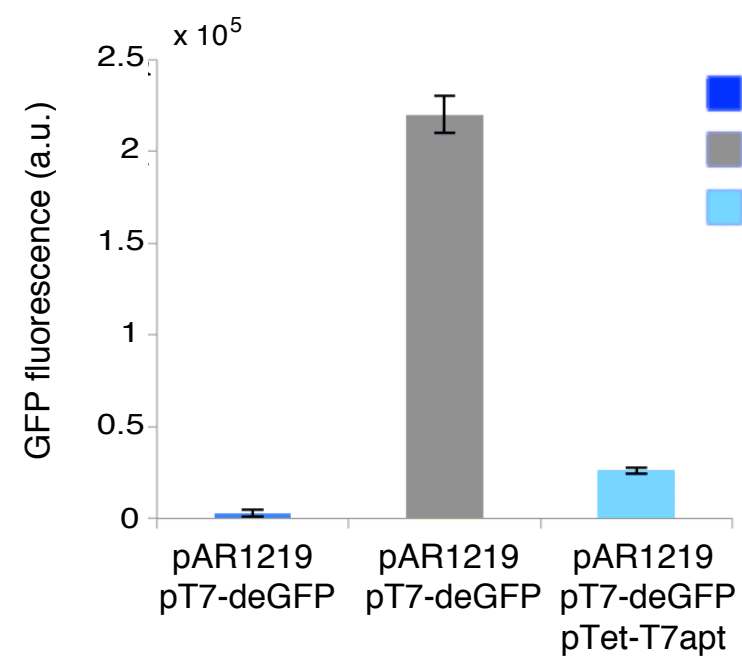

Figure S3: Genetic circuits and aptamer-mediated T7 RNAP inhibition in the TX-TL system. (A) Designed circuit for in vivo experiments. (B) Three-plasmid system used for aptamer-mediated T7 RNAP inhibition test in the TX-TL cellfree extract. (C) Experimental results showed that T7 RNAP was induced by IPTG to express deGFP and that deGFP expression was decreased by 8 fold in the presence of T7 RNAP aptamer expressing plasmid pTet-T7apt. The T7 RNAP aptamer production was not inhibited by TetR since the cell extract does not contain TetR protein. The fluorescence signal from deGFP were measured at 4 hrs. 
A

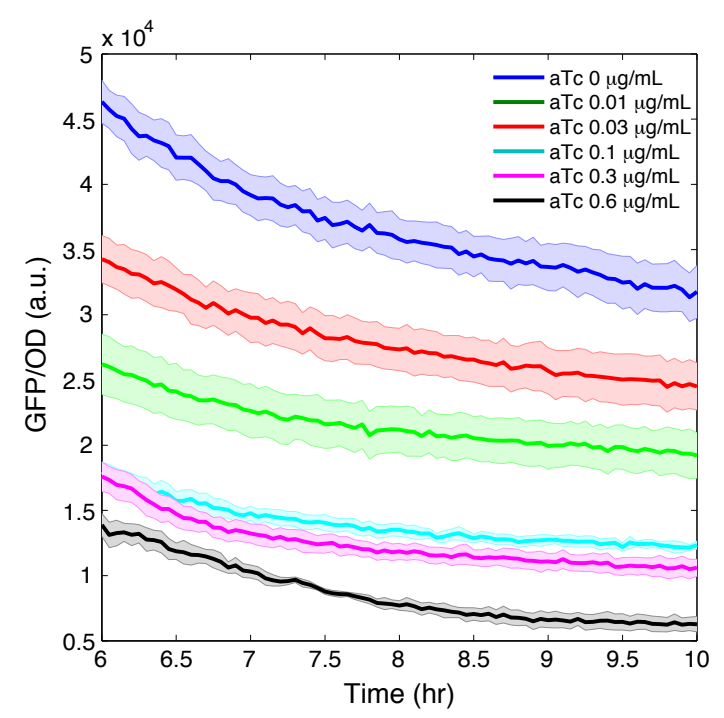

C

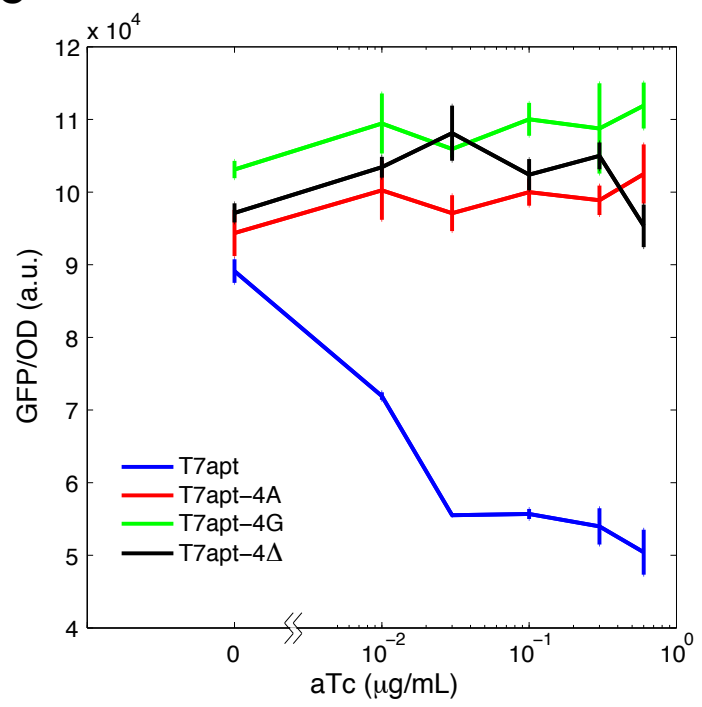

B

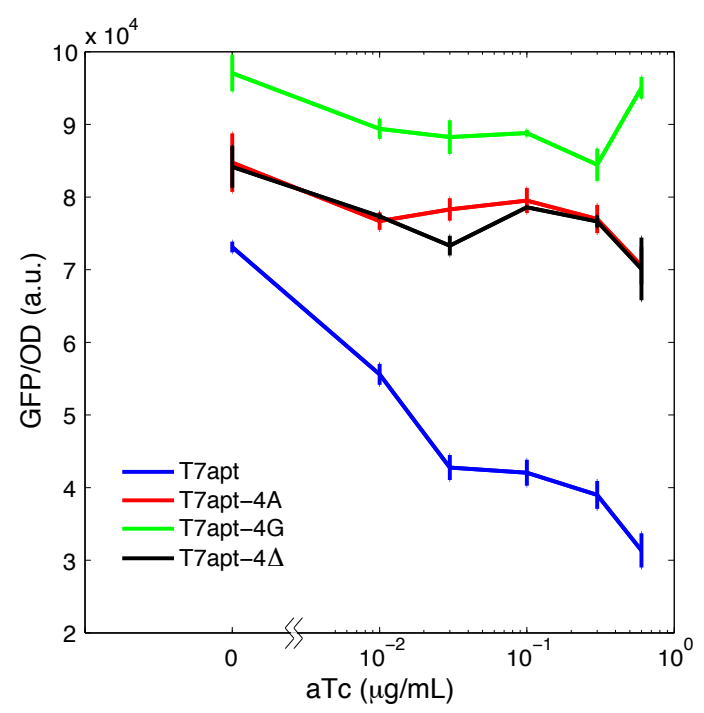

D

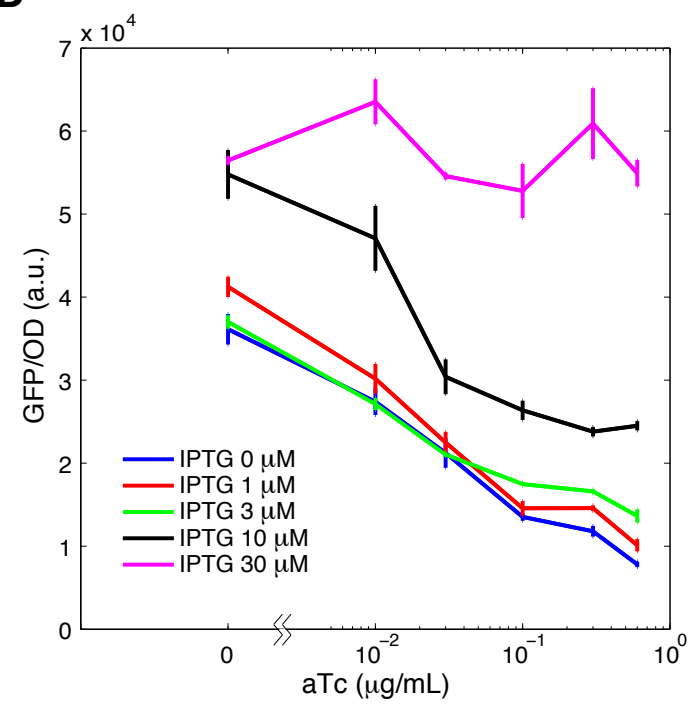

Figure S4: Plate reader analysis of T7 RNAP aptamer and variants in vivo. (A) Time course of GFP/OD measurements in LB/1\% glucose media as reported in Figure 3B left panel. The GFP/OD values decreased over time as the cells reached stationary phase after 6 hrs. However, the relative ratio of GFP/OD values for different aTc induction levels did not vary considerably during the 6 to $10 \mathrm{hr}$ time window. (B) The response curve of T7 RNAP aptamer and its variants upon aTc induction in LB media. (C) The response curve of T7 RNAP aptamer and its variants upon aTc induction in LB media with $10 \mu \mathrm{M}$ IPTG. (D) The response curve of T7 RNAP aptamer upon aTc induction in $\mathrm{LB} / 1 \%$ glucose media with different levels of IPTG. At high IPTG level, the response to aTc induction was abolished. This set of data is represented as a heat map in Figure 3B right panel. GFP/OD values were measured after 8 hrs for (B-D). 
A

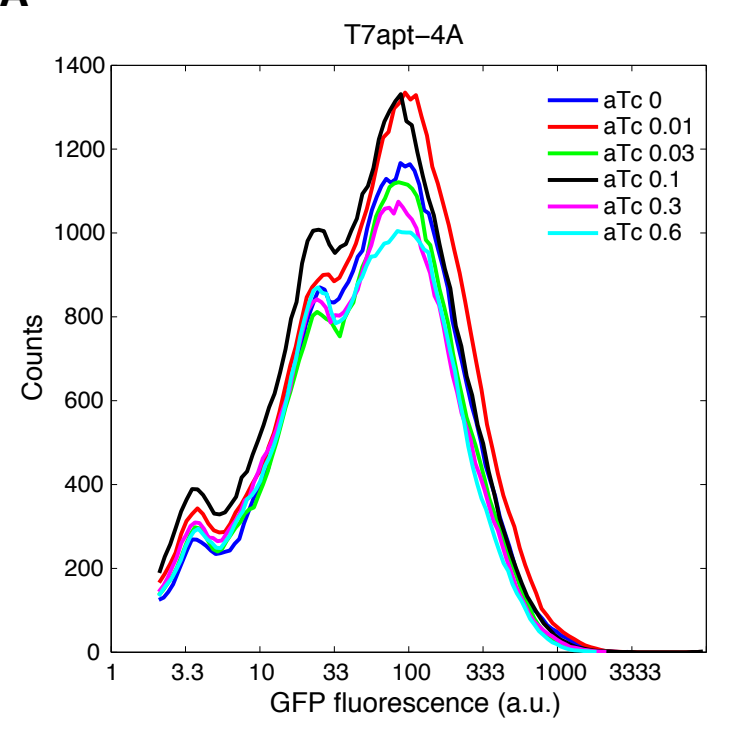

C

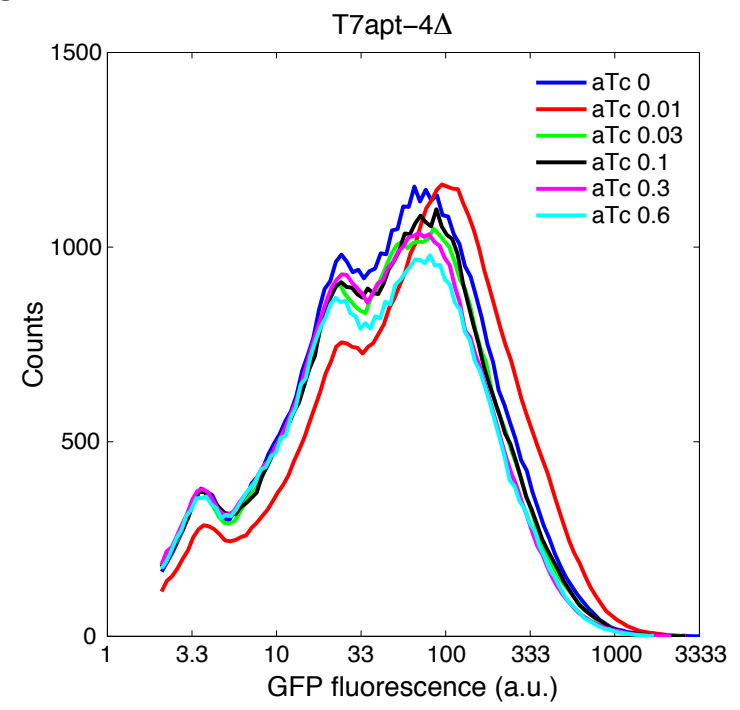

B

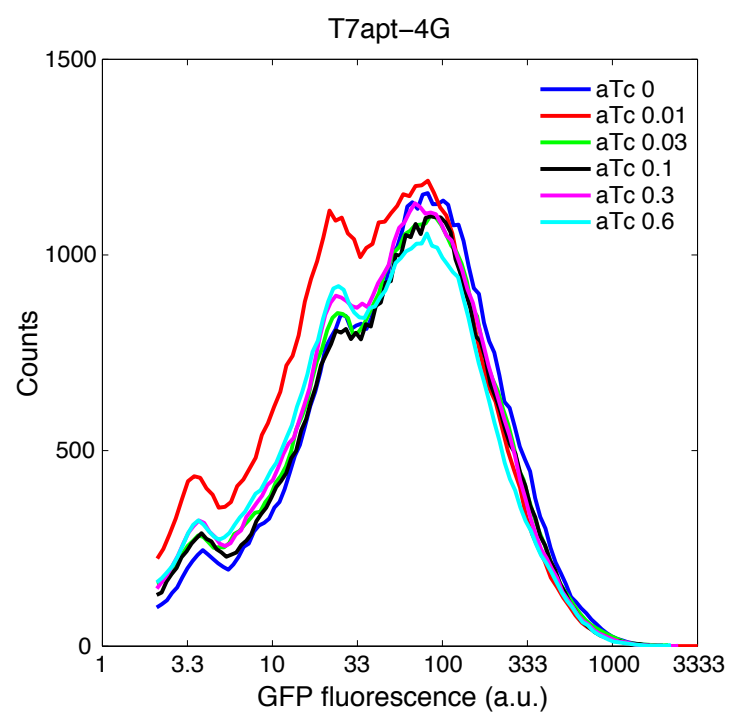

Figure S5: Flow cytometry analysis of mutant T7 RNAP aptamer in vivo. One of the replicate GFP histogram $(50,000$ events) for (A) T7apt-4A, (B) T7apt-4G, (C) T7apt-4 . The GFP expression levels did not change upon induction of T7 RNAP aptamer by aTc for all mutant aptamers. The modal GFP values are plotted in Figure 3C. 